\title{
Response inhibition is driven by top-down network mechanisms and enhanced with focused ultrasound
}

Justin M. Fine

Psychological and Brain Sciences

Indiana University

$1101 \mathrm{E} 10^{\text {th }}$ Street

Bloomington, IN 47405

Email: justfine@iu.edu

27 Keywords: Cognitive control; Response inhibition; Transcranial focused 28 ultrasound; Dynamic causal modeling; Prefrontal cortex. 


\section{Abstract}

Response inhibition is necessary for humans to safeguard against undesirable action consequences. Inhibitory control consistently recruits the prefrontal right inferior frontal gyrus (rIFG) and pre-supplementary motor area. Yet, whether inhibitory control is a defining function of rIFG, distinct from attentional orienting, remains widely debated. The issue emerges from previous studies reporting inhibitory and attentional demands both elicit rIFG activation. Here, we address this issue based on the proposition that inhibitory and attentional control are predicated on different network mechanisms. We derived and causally tested network mechanisms using EEG, dynamic causal modeling (DCM) and focused ultrasound stimulation in humans performing a Stop-Signal task. rIFG stimulation increased inhibitory performance and speed. DCM of evoked responses linked behavioral inhibition to rIFG top-down gain modulation of pre-SMA inhibitory populations. These results reconcile competing accounts of prefrontal cognitive control function, by identifying rIFG-based inhibitory mechanisms as distinct from other top-down cognitive control processes.

45

46

47 


\section{Introduction}

Response inhibition is necessary to stop unwanted actions (Logan \& Cowan, 1984). Despite its ubiquity, it remains consistently debated about whether the brain implements behavioral inhibition as a distinct cognitive control process (Aron et al., 2014; Hampshire \& Sharp, 2015; Mostofsky \& Simmonds, 2008; Munakata et al., 2011). Prominent theories consider the right inferior frontal gyrus (rIFG) a central node in driving inhibition (Aron, 2011) alongside pre-supplementary motor area (pre-SMA) (Chikazoe et al., 2009). This is supported by imaging (Aron et al., 2006; Boehler et al., 2010) indicating rIFG scales with inhibtion and pre-SMA with action preparation (Rushworth et al., 2004) and lesion studies (Aron et al., 2003). However, alternative theories posit rIFG mediates context monitoring and attention (Hampshire et al., 2010; Munakata, Herd, Chatham, Depue, Banich, \& O'Reilly, 2011). Supporting fMRI evidence indicates rIFG activates similarly across tasks involving inhibition and context monitoring, while pre-SMA activation still tracks inhibition (Erika-Florence et al., 2014; Sharp et al., 2010).

Here we propose that resolving this debate requires acknowledging that response inhibition and attentional orienting are throught to rely on different network mechanisms. Neural network models suggest response inhibition (Lo et al., 2009) could be directly implemented through top-down control over the recurrent gain of inhibitory interneurons. Indeed, neural circuits can exert inhibitory effects through feedback (i.e., top-down) connections to GABAergic interneurons (Bastos et al., 2012; Roux \& Buzsáki, 2015) that mediate disinhibition of principal neurons (Lovett-Barron et al., 2012). In contrast, biased competition (Deco \& Rolls, 2005; Desimone \& Duncan, 1995) 
80

81

82

83

84

85

86

87

and predictive coding models (Feldman \& Friston, 2010) link attentional orienting to topdown gain modulation of neurons driving bottom-up responses. Neurophysiology (Thiele \& Bellgrove, 2018) and biophysical connectivity models (Moran et al., 2013) further indicate top-down attention control over bottom-up responses coincides with gain modulation of cholinergic pyramidal cells in supragranular layers. It remains an open question whether either of these neural mechanisms can account for rIFG and pre-SMA interactions during response inhibition.

While neuroimaging studies have shown rIFG to pre-SMA connectivity scales with inhibitory success and speed (Duann et al., 2009; Rae et al., 2015), fMRI temporally filters the fast neural activity that underlies differences in response inhibition timing and success (Aron et al., 2014). Additionally, fMRI connectivity cannot differentiate top-down from bottom-up coupling or modulatory synaptic gain mechanisms. Although previous electrophysiological connectivity studies of inhibition have the proper temporal resolution, these approaches did not directly address synaptic gain mechanisms (Kibleur et al., 2016; Swann et al., 2011).

We address these gaps by combining neurostimulation of rIFG using highresolution transcranial focused ultrasound (TFUS) with electroencephalography (EEG) to examine event-related potentials (ERPs) in humans performing a Stop-Signal task (Logan \& Cowan, 1984). Several studies have shown the P300 ERP peak tracks the success of inhibitory outcomes (Bekker et al., 2005; Greenhouse \& Wessel, 2013; Kok et al., 2004). However, because P300 peaks after stop responses, this amplitude modulation occurs too late to indicate inhibition per se. Others argue P300 amplitude reflects an outcome-monitoring (Huster, Enriquez-geppert, et al., 2013) or attentional 
103

104

105

106

107

108

109

110

111

112

113

114

115

116

117 interneuron gain in pre-SMA. Imperatively, this mechanism should scale with TFUS-

118 induced effects on behavior. By modeling a network that included fronto-parietal and

119 pre-SMA to rIFG connections, this allowed addressing whether pathways associated

120

\section{Methods and Materials}

\section{Participants}

123

124

orienting process (Corbetta et al., 2008; Polich, 2007) that likely occur after inhibition.

Recent evidence implicates P300 onset latency as a candidate marker of stopping

processes because it tracks inhibition success and stopping speed (Wessel \& Aron, 2015), and modulates before stop processes elapse. As we found here that TFUS

improved stopping behavior through shortening the stop signal reaction time (SSRT), we predicted P300 onset latency would track the TFUS-induced changes in response inhibition success and SSRT.

with attention were also modulated alongside direct inhibitory mechanisms.

Healthy adults $(n=63)$ were randomly assigned to one of three experimental groups. The main experimental group received transcranial focused ultrasound (TFUS) 
125

126

127

128

129

130

131

132

133

134

135

136

137

138

139

140

141

142

143

144

145

146

147

stimulation to right inferior frontal gyrus (rIFG) $(n=25 ; 19$ males, mean age $=24.1 \mathrm{yrs}$, $\mathrm{SD}=3.2 \mathrm{yrs}$.). A second group received stimulation to the ipsilateral somatosensory cortex $(n=23 ; 15$ males, mean age $=22.4$ yrs., $S D=3.3$ yrs. $)$ and was used as the cortical site active control group (S1). A third group received sham stimulation near the right temple ( $n=15 ; 8$ males, mean age $=24.2$ yrs., SD $=2.8$ yrs. $)$ and was used as control for possible auditory effects of TFUS (sham rIFG). All individuals were righthanded (self reported) and received financial compensation for participation in the study. Before enrollment, each subject was screened for neurological disorders and a history of epilepsy, stroke, or brain injury. A neurologist from Barrow Neurological Institute (Phoenix, AZ) screened all subjects' T1 MRI and cleared them before study participation.

\section{Behavioral Task and Transcranial Focused Ultrasound design}

Response inhibition was assessed using the Stop-Signal Task involving both 'Go' and 'Stop' trials (Fig. 1) programmed in Opensesame (Mathôt et al., 2012). Each trial started with a central fixation cross. In every trial, fixations were replaced by a green 'Go' circle $\left(3^{\circ} \times 3^{\circ}\right.$ visual angle) after an exponentially-distributed time interval (range: 350-650 ms; mean: $500 \mathrm{~ms}$; standard deviation: $50 \mathrm{~ms}$ ). Subjects were instructed "to press the up key when detecting the Go circle” (Fig. 1). In 'Go' trials (rows 1-2, Fig. 1), the circle vanished either after the subject's response or 800 ms elapsed. In 'Stop' trials (rows 3-5, Fig. 1), the Stop signal was a red square which appeared around the green circle. If the subject successfully inhibited their response with respect to the Stop cue within $800 \mathrm{~ms}$, the red square was extinguished, and the trial was considered a successful inhibition. The time required to inhibit a response following the Stop signal is 
148

149

150

151

152

153

154

155

defined as stop signal reaction time (SSRT) (see below). Timing of the Stop cue relative to Go cue, i.e., the stop signal delay (SSD), was presented at one of four fixed, but subject-specific SSDs. The SSDs were chosen by having each subject perform a practice block of 50 Go trials to determine their baseline Go reaction time (RT). After this block, the 4 SSD levels were set to $25,35,75$ and $95 \%$ of the mean Go RT. These SSDs were fixed throughout the experimental session and were presented in a random order across Stop trials. All trials were separated by a 2 -s inter-trial interval $( \pm 300 \mathrm{~ms}$ random jitter).
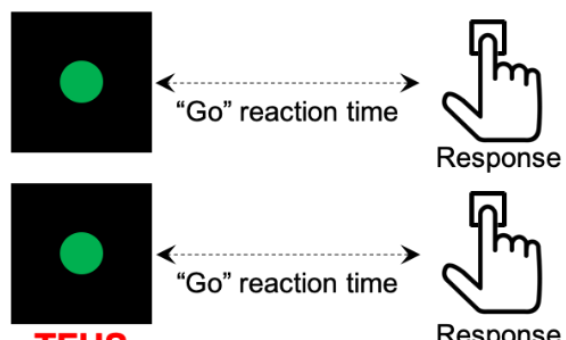

TFUS
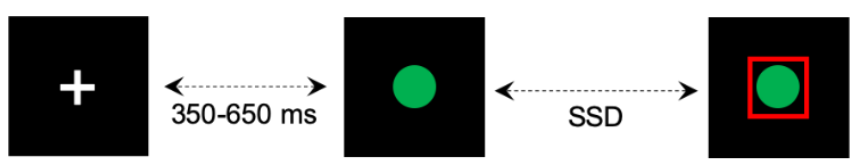

Fixation

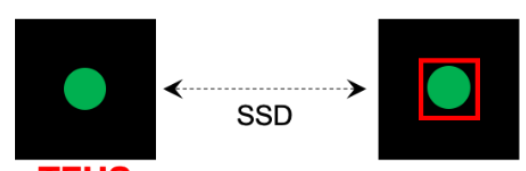

TFUS

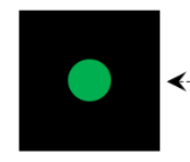

“Go" signal

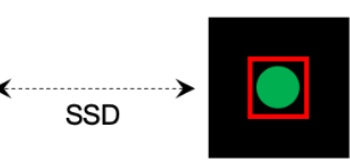

TFUS "Stop" signal

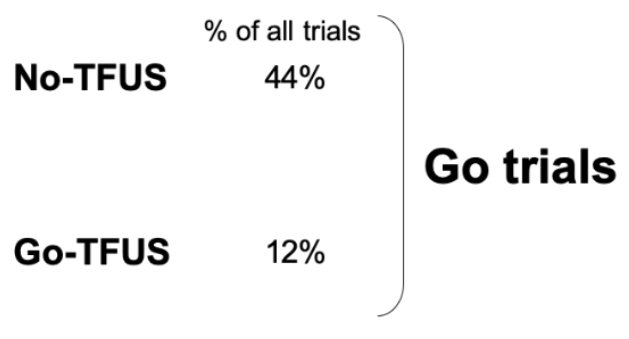

No-TFUS

$22 \%$

Go-TFUS $\quad 11 \%$

Stop trials

Figure 1 Stop-Signal task and trial types: rIFG group. Each trial type started with a fixation. After a randomly chosen delay (350-650 ms), subjects were asked to respond to a "Go" signal as fast as possible by pressing the up key. On a subset of trials (Stop trials; rows 3-5), a red square appeared at random latencies from the Go signal cueing subject to inhibit their response. Transcranial focused ultrasound (TFUS) was delivered to rIFG for 500 ms either at the onset of the Go (rows 2 and 4) or Stop signal (row 5). SSD: stop signal delay. The same design was used for two control groups ( $\mathrm{S} 1$ and sham rIFG), although TFUS was not delivered to a cortical site in the sham rIFG group (see text for details). 
We delivered Transcranial Focused Ultrasound (TFUS) either simultaneously

TFUS delivery for Stop trials was evenly distributed across the 4 SSD levels. The occurrence of Go than Stop trials (56\%; the percentage of each trial type is shown in Fig. 1). associated with TFUS were specific to the target site (rIFG). 
EEG recording. EEG was recorded using a 64-channel ActiCap system

188

189

190

191

192

193

194

195

196

197

198

199

200

201

202

203

204

205

206

207

208

209

(BrainVision, Morrisville, NC), with 10-20 layout. Data was recorded at a sampling rate of $5 \mathrm{kHz}$, with $0.1 \mu \mathrm{V}$ resolution and bandpass filter of $0.1-100 \mathrm{~Hz}$. Impedances were kept $<5 \mathrm{k} \Omega$. Online recordings utilized a ground at AFz and left mastoid reference. At the beginning of each session, electrode layouts with respect to each individual's head shape were registered using the left and right preauricular, and nasion as fiducial landmarks. This allowed for later co-registration with each individuals T1 structural MRI scan and for source-localized analysis (see below).

Structural MRI (T1). For guiding TFUS neuronavigation and co-registering EEG electrode placement for source analysis and modeling, we obtained a structural T1 MRI scan for each participant. T1 volumes were collected using an 3D MPRAGE sequence $\left(\mathrm{TR}=2300 \mathrm{~ms}, \mathrm{TE}=4.5 \mathrm{~ms}, 1 \times 1 \times 1.1 \mathrm{~mm}^{3}\right.$ voxels, field of view $240 \times 256 \mathrm{~mm}^{2}, 180$ sagittal slices) in a Philips Ingenia 3T scanner with a 32-channel head coil. Brainsuite was used to process T1s, which included cortical extraction sequence and a surface label-registration procedure with the $\mathrm{BCI}-\mathrm{DNI}$ atlas. After labeling, we checked the locations and created a mask of either pars opercularis (rIFG group) or the centroid of ipsilateral S1 (S1 group). This volume labeling and mask creation procedure were used for guiding TFUS target identification.

\section{TFUS targeting, setup and parameters}

A BrainSight neuronavigation system (Rogue industries) along with subjects' T1 scans were used to guide placement of the focused ultrasound transducer beam profile for stimulation. This was done separately with respect to each individual's neuroanatomy and mask created from T1s. The first step involved creating a subject- 
210

211

212

213

214

215

216

217

218

219

220

221

222

223

224

225

226

227

228

229

230

231

232

specific mask from cortical atlas registration and projecting into the Montreal Neurologic Institute (MNI) coordinate system. When planning the TFUS target, we considered both $\mathrm{MNI}$ coordinates and individual anatomy. For example, metanalysis studies have shown specific activation of the pars opercularis (around $x=48, y=16, z=18$ ) for contrasts of successful inhibition versus Go trials and successful versus failed inhibition trials (Chikazoe et al., 2009; Levy \& Wagner, 2011). For the rIFG group, we first identified the pars opercularis MNI coordinates. During target planning, we confirmed the coordinates were inside the anatomical region of pars opercularis. We visually confirmed each subject's pars opercularis TFUS target was rostral to the inferior precentral sulcus and dorsal to the sylvian fissure, and ventral to the inferior frontal sulcus. For the $\mathrm{S} 1$ group, TFUS was targeted near MNI coordinates of $x=-43, y=-29, z=54$ and within the left postcentral gyrus.

Before TFUS transducer setup, neuronavigation registered subjects' T1 scans in virtual space, with their head and the ultrasound transducer in real space. Alignment and cortical registration were performed using nasion, tip of the nose, philtrum, and left and right periauricular notch and tragus as fiducial landmarks. A 3D printed housing held the TFUS transducer, optical trackers, and silicon spacers (ss-6060 Silicon Solutions, Cuyahoga Falls, OH). Acoustic gel was applied to both transducer and scalp. We recorded stimulation target coordinates after placing the transducer in target alignment. In the sham rIFG group, we delivered sham TFUS (Legon et al., 2018) by placing the transducer perpendicular to the rIFG target. For the rIFG and S1 groups, we measured accuracy of stimulation target coordinates by tracking the deviation of the TFUS beam profile from the cortical target throughout the experiment. During the 
233

234

235

236

237

238

239

240

241

242

243

244

245

246

247

248

249

250

251

252

253

254

255

experimental session, we sampled TFUS transducer spatial target deviation during each break. Accuracy was very high, with an average deviation of $\pm 1.5 \mathrm{~mm}$ displacement across all subjects and sessions.

Our TFUS setup and parameters were nearly identical to those used in Legon et al. (2014). Briefly, we used a single-element TFUS transducer with a center frequency of $0.5 \mathrm{MHz}$, focal depth of $30 \mathrm{~mm}$, a lateral spatial resolution of $4.5 \mathrm{~mm}^{2}$, and axial spatial resolution of $18 \mathrm{~mm}^{2}$ (Blatek, Inc., State College, PA). TFUS waveforms were generated using a two-channel, $2 \mathrm{MHz}$ function generator (BK Precision). The system operated by channel 1 produced a pulse repetition frequency (PRF) of $1.0 \mathrm{kHz}$. Channel 1 also triggered channel 2, which produced short bursts at the $0.5 \mathrm{MHz}$ acoustic frequency. This produced an ultrasound waveform with a carrier frequency of $0.5 \mathrm{MHz}$, PRF of $1.0 \mathrm{kHz}$, and duty cycle of $24 \%$. Each stimulation duration was $0.5 \mathrm{~s}$.

Transducer power was driven by output from a 40-W linear RF amplifier (E\&I 240L;

Electronics and Innovation). It has been previously verified that the resulting waveform does not cause heating of skin or skull bone (Legon et al., 2014).

\section{Computational simulation and validation of TFUS propagation}

We quantified peak pressure amplitude, peak intensity and accuracy of the TFUS beam distribution delivered to rIFG using the pseudospectral simulation method in Kwave (Treeby \& Cox, 2010). Reference peak pressure planes for the simulations were derived from previous data (Legon et al., 2014). Simulation parameters were first validated by simulating the transducer in water to compare the simulation results with those from previous water tank tests (Legon et al., 2014). The maximum pressure plane at the $30-\mathrm{mm}$ focus was used as a source input pressure for the transducer during the 
256

257

258

259

260

261

262

263

264

265

266

267

268

269

270

271

272

273

274

275

276

277

278

simulation. The transducer was modeled to have a $30-\mathrm{mm}$ radius of curvature. For water simulations, we used a homogenously medium of water density $\left(1000 \mathrm{~kg} / \mathrm{m}^{3}\right)$ and speed of sound (1482 m/s). We created a computational grid over a $256 \times 256 \times 256$ with 1-mm spacing. The points per wavelength were 6 , Courant-Friedrichs-Lewy $=0.1$, and simulation time was set to 6 pulses (duration $=250 \mu \mathrm{s}$ ) to ensure simulation stability.

For simulating transcranial ultrasound stimulation, we extracted 3-dimensional maps of the skull from a CT (1-mm resolution) and brain from T1 MRI scans (1-mm resolution) from three preoperative patients at Barrow Neurological institute. The MRI and CT were both co-registered and normalized to the MNI space in SPM12. To mimic our approach of TFUS targeting used in the experiments, we surface registered the gray matter volume to the $\mathrm{BCI}-\mathrm{DNI}$ atlas and identified the centroid of pars opercularis. The average stimulation location for these three subjects was $x=48, y=18$, and $z=6$. This allowed us to map from world coordinates of the scan to MNI coordinates of the target.

Figure 2A shows T1 and scalp from one subject, together with renderings of the transducer housing, the pars opercularis mask, and the TFUS target applied to all MRIs from the rIFG group. Figure 2B shows side views of non-normalized T1s, pars opecularis masks, and the TFUS targets (red dots) for four subjects (Figure 2B). Conversion from Hounsfield units in the CT to sound speed and density were done using the relations described in Aubry et al (2003). All skull materials were set using these parameters, while other tissues were treated as homogeneous with parameters set to that of water. Attenuation was modeled as a power law with a $\beta=0.5$ while absorption was modeled with $b=1.08$ (Treeby and Cox, 2010). 
To assess transcranial stimulation accuracy, the simulated transcranial transmission was compared against simulations of TFUS transmission through water. absorption and change in acoustic profile after skull transmission. Numerical simulation parameters (see above) were derived to ensure the water simulation here matched the water tank results from a previous study using the same transducer and TFUS

A
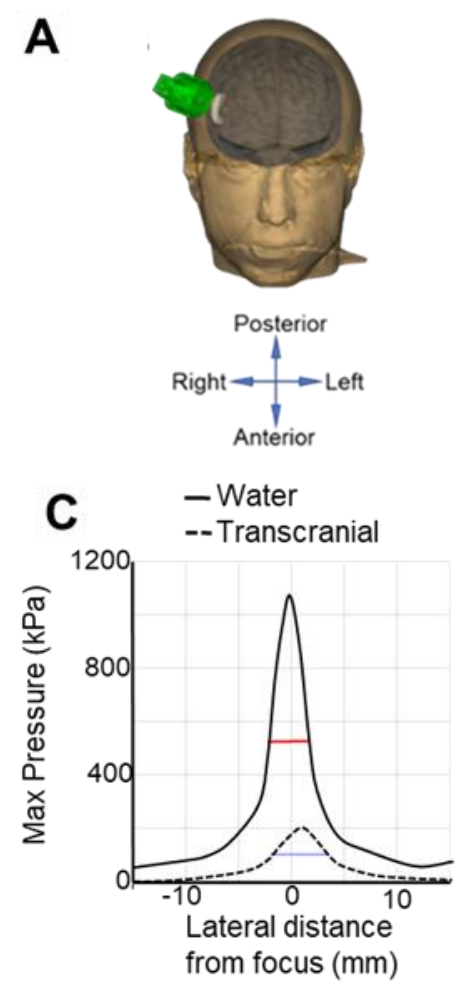

B
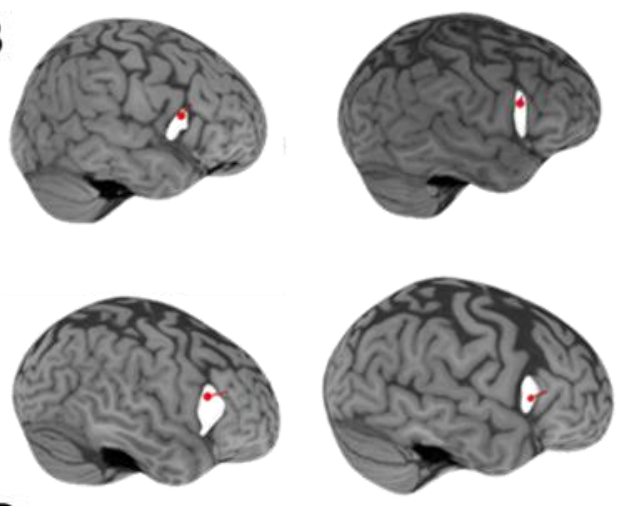

D
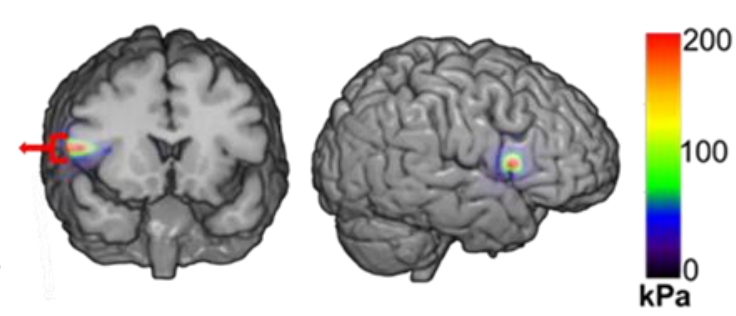

Figure 2 TFUS targeting and simulation. A. Average neuronavigation location of TFUS (red dot) applied to all MRIs used in the rIFG group. B. Structural brain scans and renderings of the targeted TFUS point (red dot) in pars opercularis (4 subjects). C. Lateral maximum pressure profile obtained at $30-\mathrm{mm}$ depth focus in both water and TFUS simulations on a CT scan from one patient (solid and dotted lines, 
respectively). Horizontal red and blue lines denote full-width half maximum of the spatial profile of lateral pressure. D. Simulated transcranial pressure profile onto T1 MRI plot shown as a color overlay. Comparison of simulations and previous water tank data (Legon et al., 2014) using the same transducer and experimental TFUS parameters indicated a $97 \%$ match of pressure/intensity at the focus taken over a $5 \mathrm{~mm}^{3}$ voxel section in all 3 planes at the focus. Next, modeling of transcranial transmission predicted an average maximum intensity of $2.8 \mathrm{~W} / \mathrm{cm}^{2}$, which is the intensity range of non-thermal neuromodulation and that exhibits nearly instantaneous measurable effects on EEG (Legon et al., 2014). Comparing the water and transcranial simulation, accuracy was assessed by comparing shifts in peak pressure. Skull transmission compared to water was shifted $1.25 \mathrm{~mm}$ laterally and had a lateral beam profile full-width half maximum of $5.1 \mathrm{~mm}$ (Figure 2C). These transcranial simulations indicate high spatial precision, with $>95 \%$ of pressure or energy $(\mathrm{kPa})$ being constrained to pars opercularis in the rIFG group (Fig. 2D).

\section{Statistical analysis}

Behavioral variables. Our behavioral analyses focused on the following variables: Go trial reaction time (Go RT), percentage of successfully inhibited responses on Stop trials (successful stopping) per SSD, failed inhibition reaction time, and SSRT. The SSRT was estimated using a hierarchical Bayesian parametric approach (Matzke et al., 2013a) that estimates distribution of SSRTs while assuming an ex-gaussian parametric form. We chose this approach as Matzke et al. (2013a) showed that it performs well even when there are only a few trials available per SSD level. This SSRT estimation procedure was run separately per group (rIFG, S1, and Sham rIFG) and trial types (No-TFUS Stop trials, Go-TFUS Stop trials, and Stop-TFUS Stop trials; Fig. 1). As 
318 we report in the Results section, we used Go RTs combined from Go trials with and without TFUS because stimulation did not alter the Go RT.

322 groups by fitting a 2-parameter logistic mixed effects model with random intercepts and

323 slopes to obtain subject- and condition-specific model parameters. $\mathrm{P}$ (respond|signal),

324 denoted as $p$, were converted to a negative $\log i t(\log ((1-p) / p))$ before fitting. As our main

325 goal was to estimate the logistic curve slope $(\boldsymbol{\beta})$, we ran the mixed-effects model (using

326 LME4 in R) with the full interaction of SSD and stimulation condition in the three Stop

327 trial types (no-TFUS, Go-TFUS, Stop-TFUS). Logistic slopes per subject were

328 estimated by combining fixed and random coefficients. $\boldsymbol{\beta}$ parameters were analyzed

329 using a mixed-design ANOVA on $\boldsymbol{\beta}$ with factors of Group (3 levels) and TFUS (3 levels:

330 No, Go, Stop).

\section{EEG pre-processing}

Continuous EEG data were first down-sampled to $250 \mathrm{~Hz}$, then high-pass filtered $(0.1 \mathrm{~Hz})$ and re-referenced to the scalp average. Any channels that displayed artifacts for more than $25 \%$ of the total session were removed before further processing, but were later interpolated after artifact rejection. We removed channels that were designated unsuitable for analysis by visual inspection and absolute temporal standard deviation ( $>5$ ) across channels. It is important to note that, in each of the stimulation groups, the cortical sites of rIFG and S1 were close to the F8 and CP4 electrodes. Therefore, these electrodes could not be used for EEG recording in their groups and were interpolated after artifact removal. Remaining data were epoched from Stop trials 
341 locked to stop signal onset (-200 to 600 ms peristimulus). Individual epochs were

342 rejected from analysis if they contained large scalp EMG or rare events $(<8 \%$ of all

343 trials). Out of the 63 participants, 3 subjects were excluded from analyses due to EEG

344 recording issues (impedance $>25 \mathrm{k} \Omega$ across channels). The remaining data were

345 bandpass filtered from $0.5-40 \mathrm{~Hz}$. EOG artifacts were removed using Independent

346 Components Analysis using eeglab (ICA; Delorme \& Makeig, 2004). On average, 3.35

347 components were removed per participant. Remaining ICA components were back-

348 projected to electrodes and then averaged to create event-related potentials (ERPs) per

349 condition, e.g., different SSD levels for No-tFUS Stop trials). The ERPs baseline were

350 normalized by subtracting the activity from $-100 \mathrm{~ms}$ to the Stop signal onset. Because

351 we later applied a Hanning taper to the edges of the ERPs before dynamic causal

352 modeling, we applied the same procedure to ERPs after cleaning using a Hanning

353 taper.

Isolating inhibitory response ERPs

Our analysis focused on sensor, source, and source network models of ERPs

because several types of ERPs have been linked to the success and speed of stopping.

357 Due to the macroscopic nature of EEG signals, there is likely a temporal overlap of neural processes related to stopping and going during Stop trials. We aimed to separate

359 Go-related activity from Stop-related activity separately for both successfully (SS) and

360 unsuccessfully (US) inhibited Stop trials. This was done by subtracting the Go trial

361 ERPs from the Stop trial ERPs, and using the approach employed by Mattia et al

362 (2012). On a per-subject basis, we found Go-trial ERPs (No-TFUS and TFUS) whose

363 RTs matched those of SS trials based on each subject's SSRT. These Go trials had to 
364 have RTs either equal to or greater than the SSRT. For US stopping trials, we found latency-matched Go trials with RTs by first calculating each subject's mean signalrespond $\mathrm{RT}$ for each of the two highest SSDs. We then calculated the difference in SSD (ms) and searched for Go RT trials for each SSD that fell within the mean signalrespond RT \pm half the difference of the SSD $(\mathrm{ms})$. This was done to prevent overlap of activity from both faster and slower Go RTs and signal-respond RTs. These steps were performed separately for the highest and second highest SSD. This procedure was

371 done separately for SS and US trials for both No-TFUS and TFUS conditions. After 372 correcting the SS and US stop trial, the corrected ERPs were averaged across the two 373 highest SSDs per subject (corresponding to $85 \%$ and $105 \%$ of mean Go RT of each subject). These ERPs were used for the remaining analysis.

\section{ERP analysis}

Sensor-space analysis. We examined ERPs at the sensors level using corrected at $p<0.01$, and performed 5000 permutations for each contrast. We used two different contrasts to analyze differences for stopping success overall and TFUS effects on ERPs. The contrasts included comparison of (1) SS -US trials collapsed over NoTFUS and Stop-TFUS conditions, (2) a TFUS effect comparison of SS (No-TFUS) - SS (Stop-TFUS) contrast, and (3) the interaction contrast of SS - US trials with No-TFUS and Stop-TFUS. The first contrast is typically used to determine which areas exhibit ERPs (or brain areas) that differentiate successful inhibition. The second contrast was 
387 and Stop-TFUS conditions. The third contrast (interaction) was used to determine how the SS-US contrast differed between No-TFUS and Stop-TFUS conditions. The second and third contrasts were the primary focus of our analyses and expected to provide the most direct indication of inhibitory-specific ERP effects. Our reasoning was as follows: if the first SS-US contrast reveals ERP activity and latencies that differentiate successful and failed inhibition, and TFUS alters the potency of inhibitory processes and ERPs that differentiate the level of successful inhibition (second contrast), the interaction contrast should isolate changes in neural response strength to Stop signals and behavioral inhibition outcomes that are jointly modulated by TFUS.

Because recent work has indicated the frontocentral (ERP) P300 onset latency, rather than peak amplitude, is related to the stopping speed (SSRT) across-subjects (Wessel and Aron, 2015), we regressed the between-subject changes in SSRT as a function of the P300 latency change between No-TFUS and Stop-TFUS. To achieve this, we computed the shift in P300 onset crossings between No-TFUS and Stop-TFUS conditions in two steps. First, we took the across-subject mean frontocentral ERP waveform in a time-window of $\pm 50 \mathrm{~ms}$ around the zero crossing. To calculate each subject's zero-crossing time, we calculated the dynamic time warping distance from the template mean ERP to the subject's ERP. Second, this distance was added to the median zero-crossing time to obtain an individual subject crossing for both the No406 TFUS- and Stop-TFUS conditions. These changes in P300 onsets were then regressed 407 against individual subject differences in SSRT between conditions. 
410 connectivity, we estimated the ERP activity in source space. This step employed a

411 multiple sparse-priors approach as implemented in SPM12. The approach estimates the

412 cortical source activity of sensor ERPs from the SS and US trials across TFUS

413 conditions. We chose this Bayesian estimation method because it has been shown to

414 have good accuracy during simulation recovery studies (Belardinelli et al., 2012).

415 Furthermore, this method focuses on finding the simple, sparse solutions given the ill-

416 posed nature of source localization. We used cortical meshes to calculate the EEG

417 forward solution using a boundary element head model based on each subject's T1.

418 Source inversion was performed over a window starting from the Stop signal up to 450

419 ms to capture activity occurring over the whole stopping period. This window of source

420 data was converted to a 3D image, spatially smoothed ( $8 \mathrm{~mm}$ full-width half maximum)

421 and interpolated into MNI voxel space for each subject and condition. This was

422 converted into a statistical parametric map (SPM) and analyzed used a flexible factorial

423 design to implement a repeated-measures ANOVA to examine the main effect of

424 inhibition success (SS or US trial). The resulting SPM was analyzed with a threshold set

425 at $p<0.005$ (peak-level, uncorrected) and cluster-wise family-wise error rate $p<0.05$.

\section{Dynamic Causal Modeling}

Dynamic causal modeling (DCM) was used to analyze different neural models of

428 effective connectivity. We applied this approach to determine the network changes

429 underlying successful inhibition and how they unfolded during successful stopping in

430 both No-TFUS and Stop-TFUS conditions. Furthermore, we used the DCM to examine

431 how different sources and their connectivity under response inhibition and TFUS 
432 conditions lead to the generation of ERPs linked to successful stopping with a focus on 433 the P300.

DCM is a source analysis that estimates how each neural source - modeled using biophysically-based neural mass models - and their connectivity relates to

436 measured ERP sensor data. DCM has now been applied in many studies to examine

437 effective source connectivity using EEG and MEG data (for overview see Moran, 2015).

438 The approach uses Bayesian source model evidence to map the estimated source data 439 to the sensor data by using the lead field generated based on each subject's T1 MRI.

440 Because DCM attempts to explain the measured data, we can compare and optimize

441 different source activity networks and connectivity structures to infer the most plausible

442 explanation for the data as is routine in modeling procedures. Additionally, because

443 DCM for ERPs derives from physiological models driving source activation and connectivity, while using a forward model mapping source to sensor space, it is valuable for differentiating how connectivity changes drive ERPs in experimental conditions.

447 the MNI locations used in the DCM were based on those found in our source analysis 448 (see Results). We used source analysis areas that have been previous linked to 449 inhibitory and attentional control (Corbetta and Shulman, 2001; Swaan et al., 2009;

450 Wessel and Aron, 2017). This analysis revealed evoked power differences during 451 inhibition in areas conforming to the right lateralized network typically found during 452 inhibition (Boehler et al., 2010; Levy \& Wagner, 2011b; Sharp et al., 2010). Locations 453 included rIOG, rIFG, rTemporal, rParietal, and pre-SMA, with the source analysis results 454 used as source locations in the DCM. The MNI coordinates employed in the DCM for 
each source included rIFG ([48,28,4]), pre-SMA $([0,24,54])$, rParietal $([40,-54,50])$, rTemporal $([52,-18,-12])$, and right inferior occipital gyrus (rIOG) $([46,-76,10])$. These locations are in strong agreement with those found in meta-analyses of inhibitory control tasks (Rae et al., 2014).

Sources were modeled with the canonical microcircuit neural mass model that has been shown to embody hierarchical Bayesian predictive coding theories of brain function (Bastos et al., 2012). This model has been used extensively to model EEG/MEG connectivity during studies of attention and stimulus prediction (Auksztulewicz \& Friston, 2015; Brown et al., 2013). The model implements source data generation as four distinct neuronal populations that include the superficial and deep pyramidal cells, spiny stellate cells, and inhibitory interneurons. Each of these layers also has recurrent inhibitory gain connections that model layer-specific inhibitory neurons. Connectivity between each population is derived from laminar-specific connectivity patterns in the cortex (Douglas \& Martin, 2004; Felleman and Van Essen, 1991; Shipp, 2007). Because connections between sources are implemented as either forward (i.e., bottom-up) or backward/feedback (i.e., top-down) connections, this allows us to distinguish how specific types of connectivity are altered during both successful inhibition and as a result of TFUS.

Central to using this DCM approach was arbitrating between neural mechanisms required for a direct inhibitory control model versus an attentional control model (see Introduction). Specifically, we were concerned with the top-down interaction between rIFG to pre-SMA and temporal cortex. Both frameworks and physiology suggest a rIFG driven top-down, activity-dependent modulation of the recurrent gain in pre-SMA. Direct 
478 inhibition models predict rIFG descending activity should alter inhibitory interneuron gain

479 in pre-SMA. Conversely, attentional models predict rIFG descending activity should

480 directly alter recurrent gain of superficial pyramidal populations that drive bottom-up

481 excitation. However, previous work proposing rIFG as driving attentional reorientation

482 does not specify what the neural target of rIFG modulation would be. For example,

483 attentional modulation could follow from rIFG modulation of temporal cortex or pre-SMA,

484 and having a chain effect on parietal areas also responsible for attentional control.

485 However, such a mechanism likely operates alongside a direct inhibition from rIFG to 486 pre-SMA. To address all of these possibilities, we considered several permutations of models in which the gain was modulated either independently of top-down activity (e.g., pre-SMA isolated gain changes), or was modulated by top-down activity from other sources (e.g., from rIFG to pre-SMA). We also considered whether these gain changes occurred in superficial pyramidal or inhibitory interneuron populations estimated within a source, and considered several different mixture variants. For example, some models had rIFG modulate inhibitory interneuron gain in pre-SMA and pre-SMA exerting gain modulation over parietal superficial pyramidal units. the ERP data (DCM ' $A$ ' matrix), determined using a series of Bayesian model selections with family-wise random effects (Penny et al., 2010) by fitting each DCM to each subject's No-TFUS successful inhibition trials in a time window spanning 0-450 $\mathrm{ms}(0=$ Stop signal onset). We chose these trials as a baseline condition to determine the 499 optimal model without effects due to TFUS. Model spaces were partitioned into several 
501 hierarchy, (3) connections from lower areas (rTemporal and rParietal) to higher areas

502 (rIFG and pre-SMA), and (4) whether exogenous gaussian bump inputs were in either

503 rIOG and rIFG, or just rIOG.

We further optimized the model again to estimate the condition effect modulation on recurrent gain and extrinsic connections ('B' matrix) that differentiated successful and unsuccessful inhibition. The optimized DCM structure were fit to individual subject's ERPs for both the No-tFUS successful and failed inhibition trials together. The effects of

508 intrinsic gain were examined over two families. The first family was modulation in superficial pyramidal versus inhibitory interneurons. The second family assessed

510 whether the post-synaptic gain effects were activity-independent or activity-dependent.

511 Activity-independent gain effects presume gain changes are independent of direct

512 activity from other areas, and it is assumed to align with local neuromodulatory

513 concentration effects (Moran et al., 2013), e.g., acetycholine or dopamine concentration.

514 In contrast, activity-dependent effects result from an area's gain alterations resulting

515 from top-down descending activity levels, hence making these gains voltage dependent.

516 All of these effects were tested combinatorically over all areas, such that some sources

517 could have superficial or inhibitory populations modulated either in an activity-

518 independent or -dependent fashion. The failed inhibition trials were treated as a

519 baseline when modeling these effects. Rather than optimizing the extrinsic connections

520 before statistical analysis, we allowed them all to modulate between conditions

521 (inhibition success) as their effective changes were tested using a hierarchical model

522 across subjects (see below). After determining the DCM gain effects, the finalized DCM 
523

524

525

526

527

528

529

530

531

532

533

534

535

536

537

538

539

540

541

542

543

544

545

were fit to individual subjects' ERPs across successful and unsuccessful inhibition trials, as well as No-TFUS and Stop-TFUS conditions.

\section{Analysis of DCM results}

To analyze the resultant condition-specific connectivity changes, we employed the Parametric Empirical Bayes (PEB) framework (Friston et al., 2016). PEB is a general linear hierarchical modeling approach, which we used to perform hypothesis testing regarding how connectivity parameters both changed on average between NoTFUS and Stop-TFUS conditions, and corresponded to scaling of stopping efficiency (SSRT). Rather than considering all possible connections, we constrained the analysis to those top-down effects involving rIFG, and pre-SMA to parietal connections. We chose this subset of connections because they correspond to the core interactions underlying our hypotheses. Once the group-level GLM parameters were estimated with respect to modulation of extrinsic and intrinsic connectivity, hypothesis testing proceeded using Bayesian model reduction of the GLM. This involves turning off/on different parameters and comparing free energy of reduced models, similar to performing model likelihood ratio tests commonly used in mixed-modeling. At a design level, we tested for mean connectivity effects differentiating successful and failed inhibition and their interaction with TFUS conditions. The interaction term was also examined with respect to SSRT and mean effects. This addresses the expectation that a connection directly related to triggering inhibition and scaling its potency should be predicted by behavior in No-TFUS trials and behavioral changes in TFUS trials. After model reduction, the reported PEB model parameters were computed through Bayesian model averaging and cutoff to effects having a $>95 \%$ posterior probability. 
546 Results

Human participants performed a Stop-Signal task and received online

548

549

550

551

552

553

554

555

556

557

558

559

560

561

562

563

564

565

566

567

568 transcranial focused ultrasound (TFUS) on a subset of Go and Stop trials (Fig. 1).

Subjects were divided into groups according to TFUS stimulation type: (1) an

experimental group that received active stimulation to right pars opercularis (rIFG), (2) a

control group that received active stimulation to ipsilateral primary somatosensory

cortex to account for non-site specific TFUS effects (S1), and (3) a second control group

that received sham stimulation to account for possible TFUS-induced auditory artifacts

(sham rIFG). Stimulation was applied online at the onset of "Go" or "Stop" signals,

separately for both Go and Stop trials (Fig. 1). We hypothesized that if rIFG drives

motor inhibition, TFUS behavioral effects would be limited to alteration of stopping

during TFUS applied simultaneously with the Stop signal.

\section{TFUS to rIFG improves stopping behavior}

We first addressed how probability of failing to inhibit responses,

$\mathrm{P}$ (respond|signal), changed across TFUS conditions (No-, Go-, and Stop-; rows 3-5,

Fig. 1) and groups, by fitting a 2-parameter logistic mixed-model to obtain response

inhibition curve slopes $(\boldsymbol{\beta})$ across subjects and TFUS conditions. Analysis of $\boldsymbol{\beta}$ revealed

only the rIFG group exhibited a TFUS-altered P(respond|signal), consisting of an

enhanced ability to inhibit reponses relative to the control groups. ANOVA results

indicated a significant Group x TFUS interaction $\left(F(2,50)=3.8, p=0.034, \eta_{p}^{2}=0.17\right)$,

and an overall effect of TFUS condition $\left(F(2,50)=11.74, p=0.002, \eta_{p}^{2}=0.29\right)$. Follow-

up one-way within-group ANOVAs across TFUS conditions showed only the rIFG group

exhibited differences across the different TFUS conditions. T-tests in this group showed 
569

570

571

572

573

574

575

576

577

578

579

580

581

582

583

584

585

586

$\boldsymbol{\beta}$ for Stop-TFUS was lower than No-TFUS and Go-TFUS conditions (both $p<0.01$ :

mean $\boldsymbol{\beta}$ 's indicating change in probability for approximately $25 \%$ change in normalized

SSD: No-TFUS $=0.35$ (0.12), Go-TFUS $=0.35(0.11)$, Stop-TFUS $=0.27(0.08))$ (Fig.

3A). Therefore, TFUS effects were limited to the rIFG group and Stop-TFUS trials.
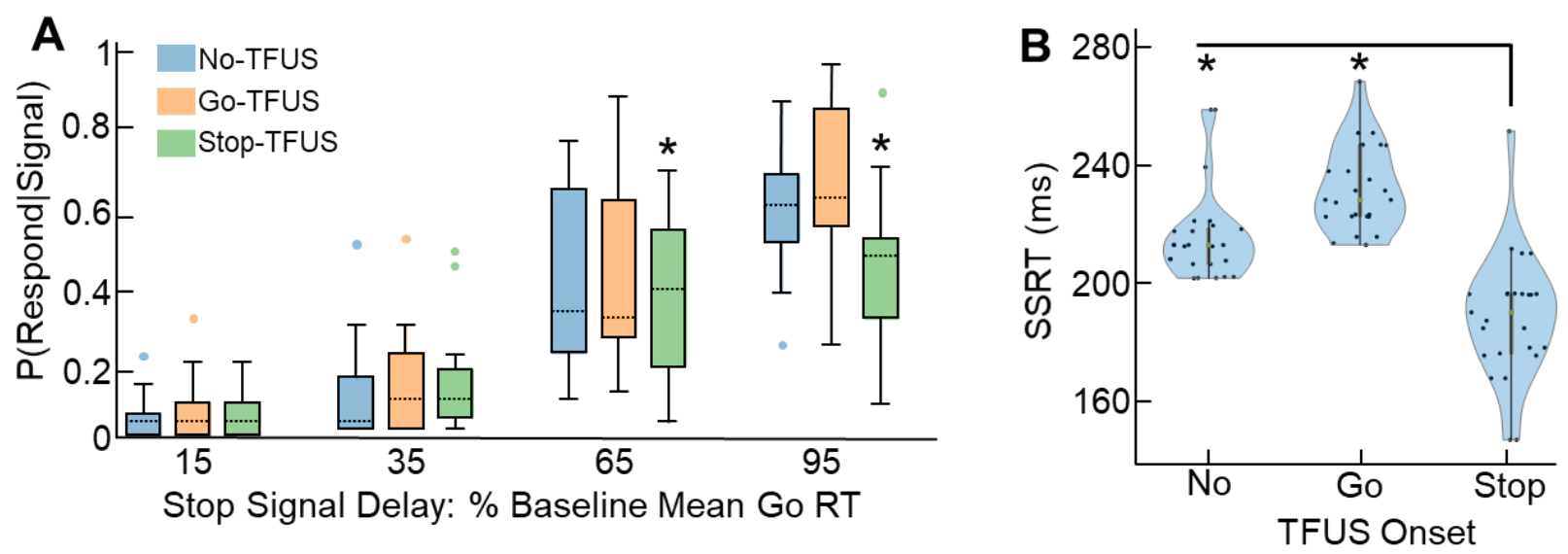

Figure 3 Behavioral effects of TFUS: rIFG group. A. Probability of responding across Stop signal delays for TFUS delivered on Stop trials. B. Violin plots of across-subject distribution of Stop signal reaction times (SSRT) across the TFUS conditions used for Stop Trials.

The effects of rIFG TFUS improvements on successful inhibition performance apper greater at longer SSDs (65\% and 95\% SSD; Fig. 3A). A repeated-measures ANOVA on P(respond|signal) for rIFG group across SSD levels and TFUS onsets supported this with a significant interaction $\left(F(6,102)=8.21, p<0.0001, \eta_{p}^{2}=0.33\right)$. T-

tests between Stop-TFUS and the average of No- and Go-TFUS across all SSDs indicated the interaction resulted from a reduction in $\mathrm{P}$ (respond|signal) for Stop-TFUS in the two longest SSDs (all $p<0.01$; Bonferroni $\alpha=0.0125$ ). These results indicate StopTFUS induced improvements of inhibition were more pronounced at longer SSDs (Fig. 3A). 
Based on the prediction that rIFG drives an inhibitory process and our finding that TFUS improved response inhibition only in the rIFG group, we hypothesized rIFG TFUS changes to $\mathrm{P}$ (respond|signal) should result from a shortening of the stopping speed, i.e., SSRT. Notably, TFUS did not affect other behavioral variables, e.g., Go RTs. SSRT analysis in a mixed-design ANOVA indicated a significant Group x TFUS interaction $\left(F(4,100)=10.2, p<0.001, \eta_{p}^{2}=0.21\right)$. Follow-up one-way ANOVAs within groups indicated only rIFG group SSRTs (Fig. 3B) significantly differed across TFUS conditions $(p<0.05)$, with t-tests confirming that SSRTs were indeed shortest and only altered for Stop-TFUS trials (Fig. 3B). This indicates TFUS delivered to rIFG concident with the Stop signal altered inhibition by shortening the SSRT. from TFUS were also affected by non-inhibitory behaviors. We first addressed whether TFUS within the rIFG, S1, and rIFG sham groups exerted any effects on simply responding to the Go signal by extracting mean $\mathrm{RT}$ from ex-gaussian distributions fit using maximum likelihood (Lacoutoure and Cousineau, 2008). Means for each subject were analyzed using a $3 \times 2$ mixed-design ANOVA with factors of Groups (3) and TFUS condition (2: No-TFUS, Go-TFUS trials). This analysis allowed us to assess if "going", 604 independent of "stopping", was altered by potential TFUS auditory artifacts (sham rIFG 605 group), stimulation to unrelated areas (S1 group), or whether TFUS to rIFG also influenced Go RT processes independent of a stopping context (rIFG group). We found 607 no significant effect of TFUS condition, group, or their interaction (all $p>0.05$ ). These results suggest that neither TFUS (rIFG and S1 groups) nor auditory factors alone 609 (sham rIFG group) altered Go RTs independent of a Stop signal. 
Improvements in inhibition from TFUS could also have emerged from longer

611 SSRTs or reduced SSRT variability. Two mixed-design ANOVAs were used to examine

612 the subject-level SRRT means and variability with Group (3 levels) and TFUS (3 levels:

613 No-TFUS, Go-TFUS, Stop-TFUS). We found no significant interactions or effects of

614 TFUS on SRRT or its variability. Together, these behavioral results indicate that only

615 TFUS to rIFG improved response inhibition by shortening a process related to the 616 stopping speed.

\section{Neural responses underlying inhibition}

We will now focus only on the rIFG group because this was the only group exhibiting behavioral TFUS effects. Furthermore, we only analyzed No-TFUS and StopTFUS conditions because Go activity was subtracted from neural data from Stop trials

621 (see Methods). Sensor-level ERPs were examined in this group across three contrasts

622 using cluster-based permutation t-tests: (1) successful - unsuccessful stopping contrast

623 over each TFUS conditions, (2) successful (No-TFUS) - successful stopping (Stop-

624 TFUS) contrast, and (3) interaction comparing successful - unsuccessful stopping

625 between the No-TFUS and Stop-TFUS conditions. The average activity of ERP time-

626 windows and voltage deflections of an N200 and P300 that are typically found during

627 inhibition (Kenemans, 2015) are shown in Figure 4A. 


$$
\begin{aligned}
& \text { SS }=\text { Successful Stop } \\
& U S=\text { Unsuccessful Stop }
\end{aligned}
$$
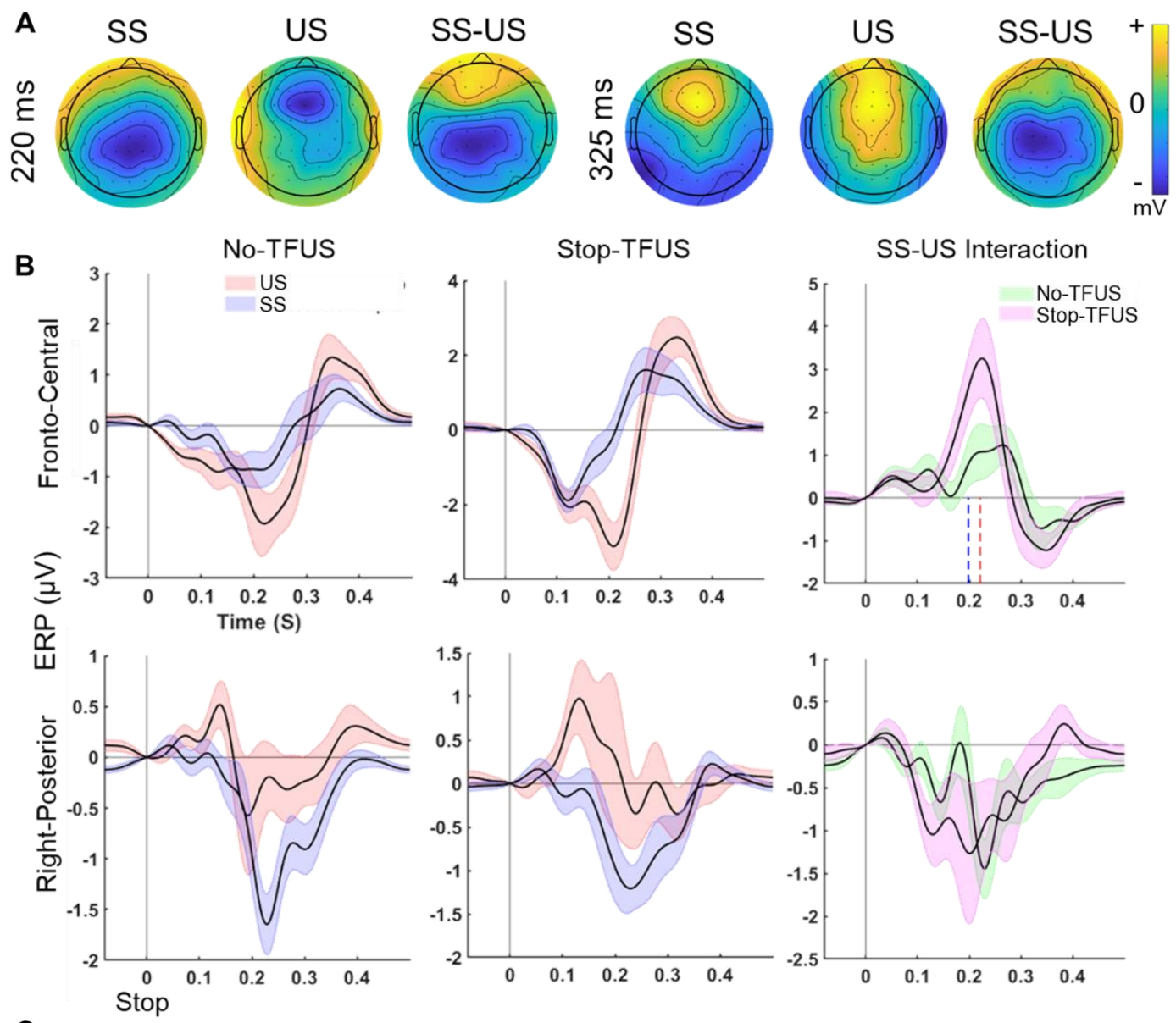

C

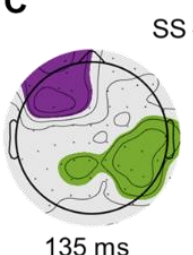

SS - US
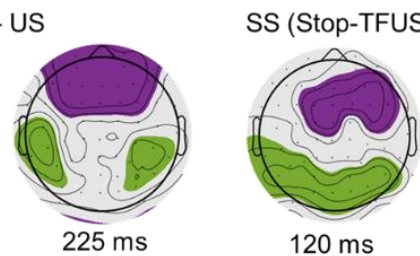

$120 \mathrm{~ms}$
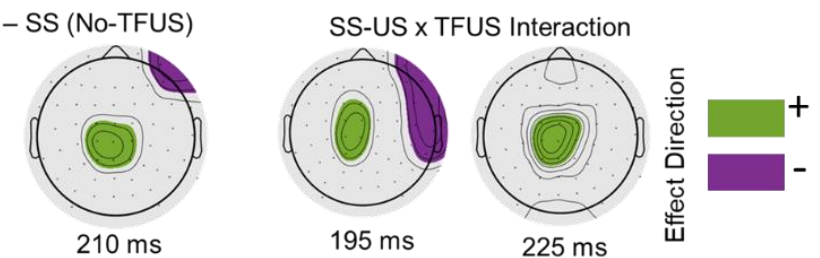

Figure 4 Effects of TFUS on ERPs during successful and unsuccessful response inhibition. A. Average scalp maps of successful (SS), unsuccessful (US), and SS-US difference maps showing ERP amplitude at time points aligned with canonical ERP effects associated with response inhibition. B. Average ERP plots for two a-priori chosen regions of electrodes to capture the typical N200/P300 ERP in Fronto-Central electrodes (Cz and Fcz; top row), and N100 sensory responses in Right-Posterior (CP4 and P4; bottom row). Plots show mean ERPs for SS and US trials across TFUS conditions (left and middle columns) and their interaction (right column). In the right column, the dashed vertical lines denote the median SSRT across subjects for the No-TFUS (red line) and Stop-TFUS (blue line) conditions under the interaction in Fronto-Central electrodes (top row). C. Permutation sensor t-tests showing the maximal 640 effects for three comparisons: SS-US, SS trials across TFUS conditions, and interaction effect of SS-US and TFUS. 
641

642

643

644

645

646

647

648

649

650

651

652

653

654

655

656

657

658

659

660

661

662

663

We observed an early (100-150 ms) right-posterior P100 response peaking around $135 \mathrm{~ms}$ that differentiated successful from unsuccessful stopping (SS and US, respectively; Figure 4B, left plot of Figure 4C). Peak location and timing are indicative of a visual P100, visible in average time-course and t-stat map (Figure 4B-C), with larger peak amplitudes in US trials. Contrasts of SS trials across TFUS conditions revealed the P100 was smaller on Stop-TFUS SS trials (Figure 4C, middle). A difference in SS trials was found for N100 responses in both frontal and right-frontal electrodes (Figure 4C, middle). N100s were generally larger for the Stop-TFUS trials. Neither of these ERPs exhibited an interaction of SS-US and TFUS trials. These results indicate smaller amplitudes of early sensory responses predicted successful stopping, but the lack of interaction indicates they were not directly related to inhibition.

The ERPs typically associated with indexing inhibition is the N200/P300 complex. Notably, this complex often appears in a fronto-central cluster, with the N200 peak being most prominent during failed stopping. The fronto-central ERP plot shows the N200 peaks around $200 \mathrm{~ms}$ but does so most clearly in US trials (Figure 4B, top row). Comparing SS and US trials supported this effect of larger N200 during US trials (Figure 4C, left). Contrasting SS trials across TFUS conditions indicated this ERP was larger for the No-TFUS condition. The interaction effect (Figure 4C, right) indicated the maximal difference corresponded to N200 timing, exhibiting a larger difference in the SS-US contrast during Stop-TFUS trials. N200 amplitude decreased with increasing inhibitory performance, with the smallest peak in Stop-TFUS SS trials (Figure 4B, top row). The fronto-central P300 also differentiated SS and US trials, with a lower 
664 665 666 667 668 669

670

671

672

673

674

675 676

amplitude for US trials. The lack of interactions for the P300 indicated it was distinct from driving the strength of inhibition.

Our main ERP hypothesis was that P300 onset timing, rather than its peak, reflects an inhibitory process. This hypothesis stems from two considerations. The first is the N200 and P300 likely reflect a mixture of underlying components with the N200 amplitude being reflected in the P300 onset. In this case, a shift in P300 onset would drive our observed interaction contrast of the fronto-central N200 by altering the time of the N200 peak amplitude. Examination of the time course of fronto-central ERPs (Fig. 4B, top row) indeed indicated that reduction in N200 amplitude is likely driven by the P300 onset occurring earlier. The second considerations is that an inhibitory marker should track inhibition timing (SSRT), which has been previously reported for P300 onsets (Wessel \& Aron, 2015). Therefore, we predicted that the P300 onset latency should correlate with the SSRT difference across TFUS conditions. Visually contrasting SS-US difference waveforms across TFUS conditions (Fig. 4B, upper right) revealed P300 onsets shifted earlier during Stop-TFUS. We found TFUS-induced changes in P300 onset significantly correlated with SSRT $(0.61, p<0.05)$, thus providing direct support of P300 latencies tracking inhibition speed.

\section{Dynamic Causal Modeling of ERPs in the inhibition network}

The combined effects of TFUS on behavioral and evoked responses indicated inhibition performance improved together with a shortening in SSRT, which was matched by a shorter P300 onset latency. Given TFUS to rIFG altered inhibition, a core question is to what extent rIFG network interactions drive these evoked responses and associated behavioral changes. We addressed this by modeling evoked responses 
687

688

689

690

691

692

693

694

695

696

697

698

699

700

701

702

703

704

705

706

707

708

709

using Dynamic Causal Modeling (DCM) to determine which connections differentiated stopping outcomes and scaled with SSRT across TFUS conditions. We considered a restricted network of areas that have consistently been found to be activated during inhibition. These areas include a fronto-parietal and cingulo-opercular network, centered around rIFG and pre-SMA, right temporal (rTemporal), right inferior occipital gyrus (rlOG), and right parietal (rParietal) areas (Levy \& Wagner, 2011). To derive DCM locations for these sources, a source analysis on the mean difference between SS and US ERPs was performed in the 0-450 ms window. Source maps (Fig. 5A) revealed areas typically associated with successful inhibition. Maximal amplitudes of these source locations were used in the subsequent DCMs (see Methods).

We used a series of Bayesian model selections with family-wise random effects to determine the optimal structure by fitting different models to sensor responses during No-TFUS successful and unsuccessful inhibition trials from all subjects. The winning family for frontal hierarchies (Fig. 5B, top) included recurrent connections between rIFG and pSMA, with rIFG having top-down (backward) connectivity to pre-SMA (exceedance probability: 0.97). The family analysis examining the lower hierarchy (Fig. 5B, bottom) revealed rIOG was, as expected, connected to rTemp and rPC, but these two areas were not laterally connected (exceedance probability: 0.89 ). Finally, the comparisons examining lower-to-higher areas connections recapitulate known ventral and dorsal pathways (exceedance probability: 0.92). rIFG had top-down and bottom-up connectivity with rTemp, and pSMA had top-down projections to rPC and forward connections to pSMA. Finally, we found the best model to have inputs to both rIOG and rIFG (exceedance probability: 0.88 ). 
A
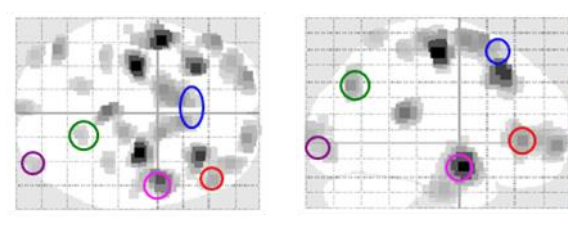

OR. Inferior Occipital (rIOG)

OR. Temporal (rTemp)

OR. Parietal (rPC)

OPre-SMA (pSMA)

R. Inferior Frontal (rIFG)

B

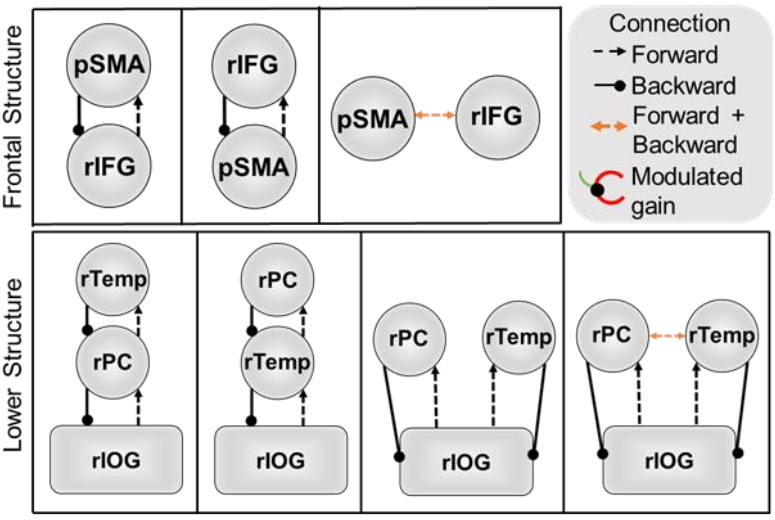

C Winning Model

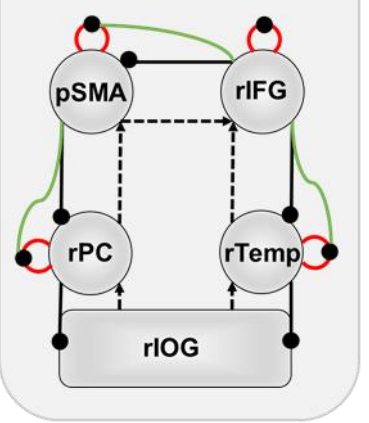

D

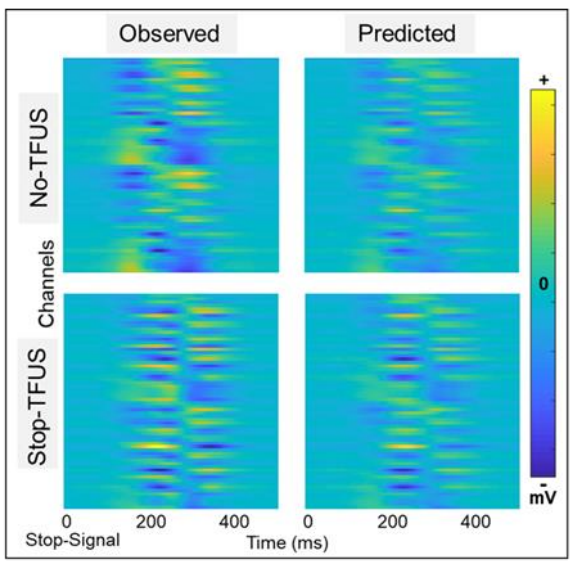

E

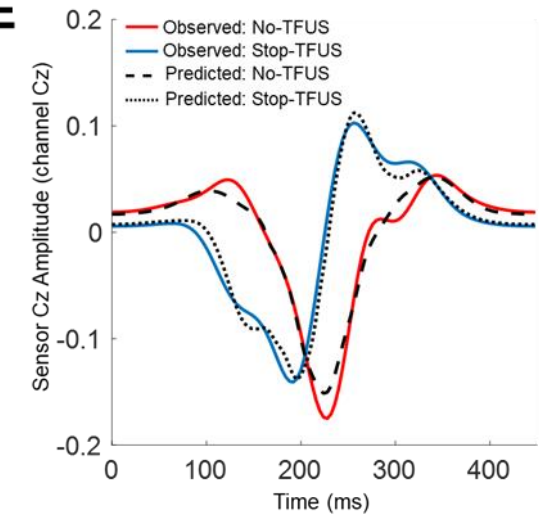

Figure 5 Dynamic Causal modeling: Sources, structure, and fit. A. Source map of grand mean ERP differences of SS minus US trials from 0 to $450 \mathrm{~ms}$. Colored circled nodes denote center of node coordinates used for the 5 regions in the DCM. B. Configurational structures used to build base DC models and their connection types. C. Winning model showing the final configuration fit to individual subjects' data. D. Observed and predicted ERPs for all EEG channels for the average of No-TFUS and Stop-TFUS trials. E. Mean predicted (black dashed and dotted lines) and observed (red and blue lines) ERP from channel Cz plotted for successful Stop trials across No- and Stop-TFUS conditions, respectively. This plot shows that the modeled ERPs captured the TFUS-induced differences in N200 and P300 onsets and amplitudes.

\section{Cortical control of stopping involves top-down rIFG gain control}

A final set of model comparisons examined permutations of the above model with modulation of postsynaptic (recurrent) gain. We considered models wherein gain 
724 changes occurred in either superficial pyramidal or inhibitory interneuron populations,

725

726

727

728

729

730

731

732

733

734

735

736

737

738

739

740

741

742

743

744

745

746

and whether the post-synaptic gain effects were activity-independent or -dependent,

and modulated by top-down activity. Model selection indicated strong evidence for a model with gain changes in inhibitory interneuron layers (probability: 0.96), all these effects being activity-dependent (probability: 0.93). Therefore, evoked activity during stopping was best explained by a network including gain changes that were dependent on top-down rIFG activity. The winning DCM used for further analysis is shown in

Figure 5C. The core result from these model tests is that, although areas such as pSMA, rPC and rTemp are involved in generating inhibition-related ERPs, the network involved top-down, activity-dependent gain modulation from rIFG.

The optimized DCM structure (Fig. 5C) was fit to individual's evoked responses as separate models for the TFUS conditions. Fitted models accurately captured spatiotemporal properties of the ERP data across both sets of model fits (Fig. 5D) with an average fit of all DCM models of $R^{2}=92 \%( \pm 9 \%)$. Figure $5 E$ shows the model faithfully captured characteristics of the N200 and P300 ERPs. The models were statistically analyzed in a parametric empirical Bayesian (PEB) model to determine which connections were modulated between SS and US trials and TFUS. As our hypotheses considered top-down coupling from rIFG and the effects of neurostimulation, for brevity we only consider these connections.

\section{rIFG connections directly modulate with stopping outcomes}

The main hypothesis was that rIFG regulates stopping via top-down gain modulation of inhibitory populations in PSMA. We predicted this modulation should be larger during successful inhibition. Model averaging over parameters corroborated this 
747 hypothesis. Inhibitory interneuron gain modulation within $\mathrm{IIFG}$ and the rIFG to pSMA connectons increased during successful inhibitory trials. The direction of these effects for connections to fronto-parietal attention areas were reversed. During US trials, the rIFG to rTemp and pSMA to rPC gain was larger (Fig. 6A). interactions of SS-US and TFUS on connectivity. The interaction of these parameters with TFUS were found in all of these connections. Intrinsic changes in rIFG gain, rIFG to pSMA, and pSMA to rPC gain modulation all exhibited interactions wherein the difference between US and SS trials was larger for Stop-TFUS trials (Fig. 6B). In contrast, the rIFG top-down modulation to rTemp was larger in the No-TFUS conditions.

757 These effects point to an increased top-down modulation of rIFG connections and cascading onto rPC. In addition, the decreasing effect from rIFG to rTemp indicates stopping propensity was associated with a reduction of top-down coupling in the ventral pathway. interneurons, this effectively increases top-down inhibition over pSMA through rIFG. A

763 similar interpretation holds for the modulation of pSMA to rPC. To elaborate, increases

764 (decreases) in self-recurrent gain of inhibitory interneurons have the effect of reducing

765 (increasing) overall inhibition in the target area. Although several parameters correlated with change in behavioral inhibition, we further assessed the predictive validity of these

767 connections as mediating stopping outcomes. This was tested by classifying the two 768 stimulation conditions using a logistic regression, where the gain connections indexing 
771

772

773

774

775

776

777

778

779

780

781

782

783

784

785

786

787

788

10 k-fold cross-validation. Only the rIFG to pSMA (66\% classification accuracy, $p<$

$0.05)$ and the pSMA to rPC connection (77\% classification accuracy, $p<0.01)$ were accurate predictors of TFUS conditional classes. Given the multiple top-down effects going from rIFG to $\mathrm{PSMA}$ and $\mathrm{rPC}$, we reasoned the $\mathrm{PSMA}$ to $\mathrm{rPC}$ change was partially dependent on effects from rIFG to pSMA. We tested this by partialing effects of rIFG-topSMA connections on the latter with a linear regression, then re-running the classification using residuals. The PSMA to rPC classification accuracy was substantially reduced, but still significant $(69 \%, p<0.01)$.

\section{rIFG connectivity modulates processing of stopping speed (SSRT)}

The core behavioral finding was TFUS improved stopping by shortening SSRT.

Accordingly, we predicted that if rIFG directly controlled inhibitory processes, its outgoing connectivity should reflect changes in SSRT across TFUS conditions.

Connections were examined by comparing rIFG connectivity between SS trials with the SSRT difference as a covariate. Using No-TFUS trials as a baseline, we found the intrinsic rIFG gain, the rIFG-to-pSMA and pSMA-to-rPC inhibitory interneuron effects scaled with the difference in SSRT (Fig. 6C). Direction of these effects specifically reflected a decreasing gain from No-TFUS to Stop-TFUS, or a larger top-down inhibition scaling with shorter SSRTs. The core issue was reversing the problem and addressing whether any of these connection changes predicted the change in SSRT. 
A
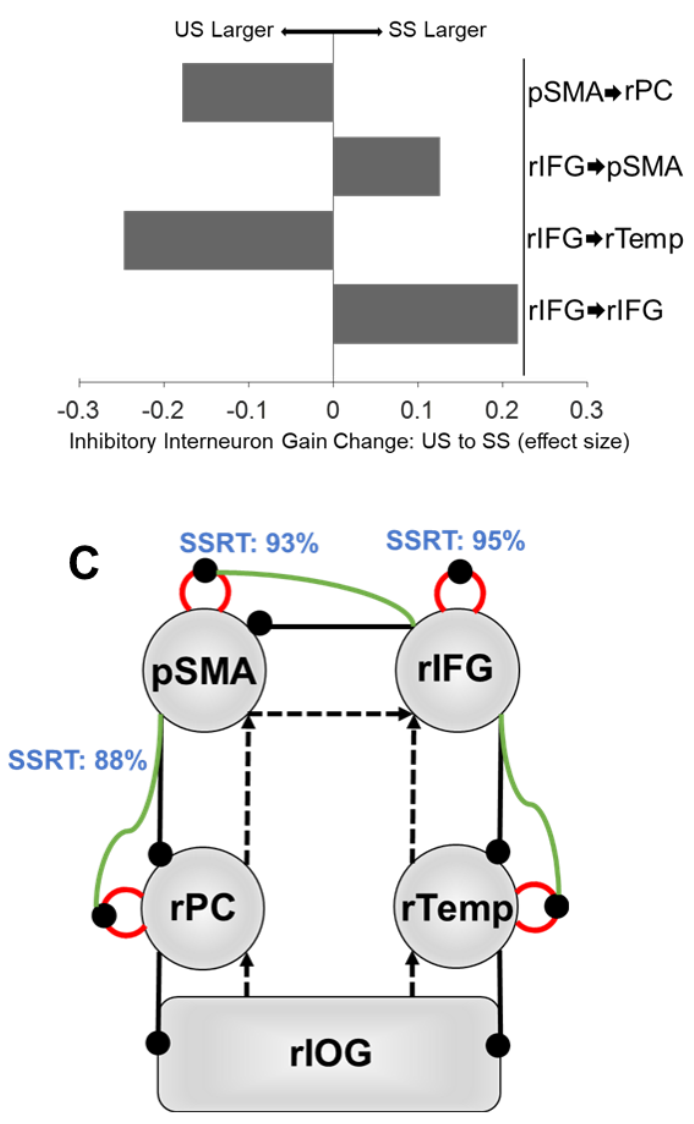

\section{B}

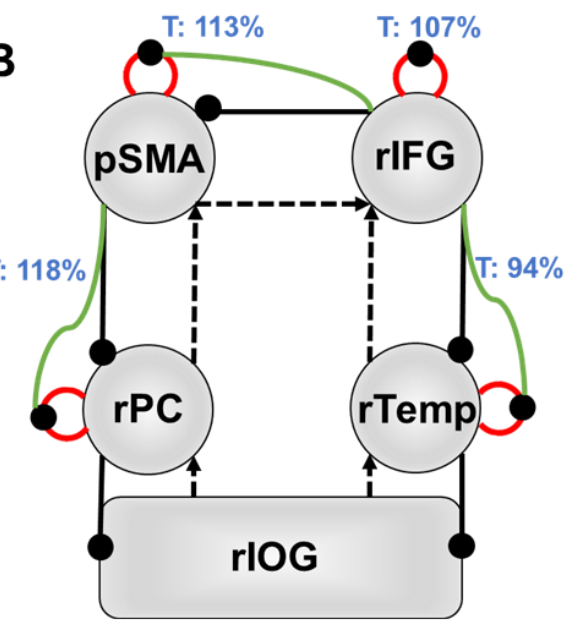

D

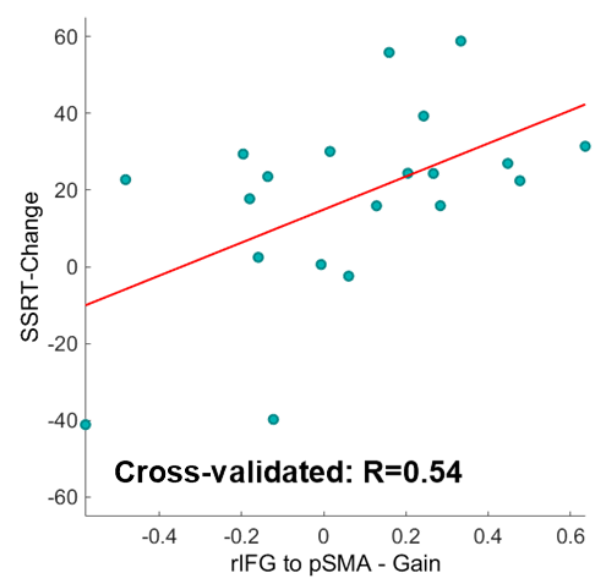

Figure 6 Parametric empirical Bayesian analysis of Dynamic Causal models to distinguish overall success of stopping versus modulation with SSRT. A-B. Results of PEB models for mean gain changes between SS and US trials in rIFG and their interactions with TFUS. C. PEB result showing parametric scaling of difference in SSRT between TFUS conditions with gain change between TFUS conditions for SS trials. D. Leave-one out cross-validated linear regression using rIFG to pre-SMA gain between (across TFUS conditions) SS trials as a predictor for the SSRT change.

We assessed the predictive strength for this SSRT effect using a leave-one out crossvalidated robust linear regression. Supporting the hypothesis that rIFG-to-pSMA topdown gain modulation drives inhibition, we found it was the only significant predictor of this effective change in SSRT ( $R=0.54, p=0.013$; Fig. 6D). 
Beyond the connectivity modulation across TFUS conditions, our scalp analysis

803

804

805

806

807

808

809

810

811

812

813

814

815

816

817

818

819

820 821 coupling driving response suppression through a direct inhibitory mechanism that

822

isolated P300 onset timing as a key marker of inhibitory outcomes and SSRT. A key

question is: Where do the P300 effects emerge from, in terms of the local rIFG gain and its top-down inhibitory modulation over pre-SMA? The connection between scalp and source activity are visible in estimated deep pyramidal source activity for both areas

(Fig. 7). We focus on deep layer activity because its strong correspondence with scalp ERPs. Timing differences in rIFG activity peaks reflected differences in stopping outcomes and SSRT. These behavioral effects were linked to a larger amplitude and earlier onset in rIFG (Fig. 7, top), respectively. As Figure 6 shows, the success and its effective increase during TFUS conditions was associated with larger rIFG interneuron self-gain or increased disinhibition. The result is a larger deep pyramidal activity and top-down inhibitory modulation over pre-SMA. Figure 7 (bottom) also shows the N200/P300 ERP complex was localized to pre-SMA. Comparing activity across areas indicated timing effects corresponding to SSRT were predominant in rIFG compared to pre-SMA, whereas pre-SMA exhibited a strong amplitude interaction across TFUS conditions. Together, these observations indicate rIFG activity reflected both inhibitory behavioral outcomes and neural response timing. The rIFG activity also points to P300 timing observed at the scalp as resulting from direct rIFG influence, rather than preSMA. Together, these results provide strong empirical support for top-down rIFG directly links to timing of scalp P300 responses. 

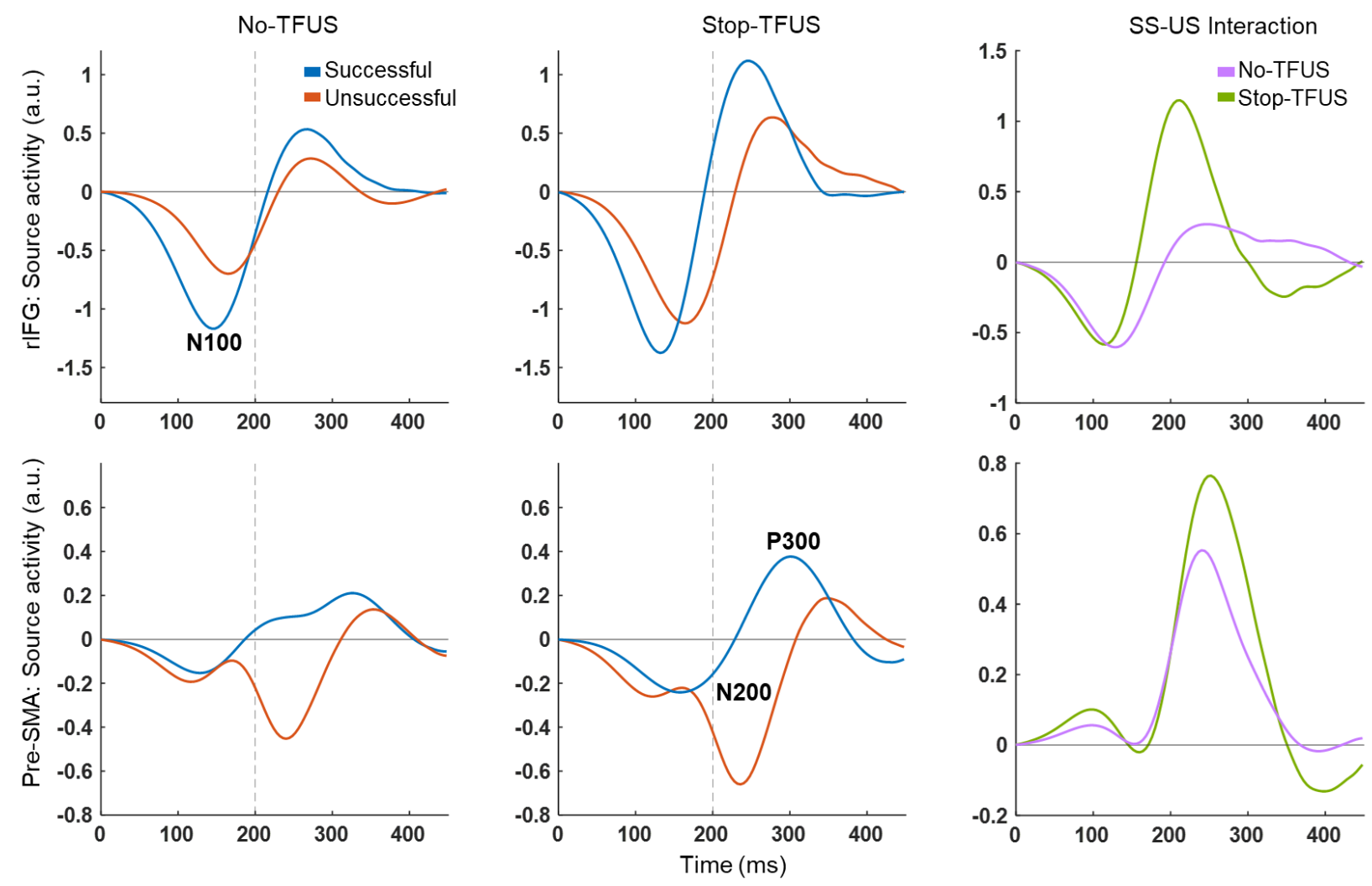

824

825

826

827

828

829

830

831
Figure 7 rIFG source activity reflects inhibitory success and timing. Top and bottom row plots show the estimated deep pyramidal source activity from rIFG and PSMA, respectively. The left and middle column plots show the comparison between successful and unsuccessful trials in the No-TFUS and Stop-TFUS conditions, respectively. The right column plots show the interaction between successful and unsuccessful trials (SS and US, respectively) in the NoTFUS and Stop-TFUS conditions.

\section{Discussion}

We provide causal evidence that rIFG directly drives response inhibition in the cortical network including pre-SMA through an inhibitory mechanism linked to ERPs.

We demonstrated this relation by applying online transcranial focused ultrasound (TFUS) to human pars opercularis portion of rIFG during a stop-signal task. TFUS

improved inhibitory performance with a faster stopping speed (SSRT) without impacting Go responses (Fig. 3). 
We first sought to determine if an event-related potential (ERP) represents inhibitory components and could be isolated using TFUS. Inspired by correlative work (Wessel and Aron, 2015), EEG analysis revealed the P300 ERP onset tracked the SSRT and inhibitory success across TFUS conditions, supporting a causal connection to behavioral inhibition (Fig. 4). Our second goal was determining if the rIFG and preSMA network interactions exhibit the top-down inhibitory gain effects predicted by neural network models, while offering a causal explanation for P300 timing effects. Our approach of using Dynamic Causal Modeling (DCM) to test specific mechanisms driving evoked responses significantly extends previous findings. The modeling pointed to response inhibition being associated with a top-down rIFG modulation of inhibitory interneuron gain within pre-SMA (Figs. 5-7). To our knowledge, these findings provide the first demonstration of a causal link between rIFG and behavioral inhibition outcomes and potency, while simultaneously isolating the core neural markers and their basis in a mechanistically-specific neural inhibitory interaction in the rIFG and pre-SMA stopping network. response inhibition from nearly all aspects. By employing online TFUS in parallel with EEG, we isolated P300 onset latencies as the sole predictor of behavioral outcomes response inhibition performance and SSRT - during No-TFUS and Stop-TFUS trials.

857 This result are consistent with recent work suggesting P300 latencies predict outcomes and SSRTs (Huster, Enriquez-Geppert, et al., 2013a; Wessel \& Aron, 2015), with

859 latency modulation occurring before but close to SSRT. This close temporal proximity of 
861 non-human primates (Hanes et al., 1998), as well as neural network (Lo et al., 2009)

862 and accumulator models (Boucher et al., 2007; Logan et al., 2015). However, our work

863 is the first to directly stimulate rIFG online and show a concurrent change in P300 onset

864 and behavior. Consistent with other studies (Bekker et al., 2005; Kok et al., 2004), we

865 also found the peak amplitudes of a posterior P100 and fronto-central N200 and P300

866 differentiated inhibitory outcomes. These effects were limited to No-TFUS trials,

867 suggesting they reflect non-inhibitory processes, e.g., sensory detection (N100;

868 Kenemans, 2015; Lijffijt et al., 2009), surprise or conflict (N200; Alexander \& Brown,

869 2011; Enriquez-Geppert et al., 2010), or outcome-monitoring and attentionally-

870 reoriented processing that could occur after the SSRT (P300; Corbetta et al., 2008;

871 Polich, 2007).

It is worth noting the N200/P300 complex is regularly source localized to pre-

873 SMA (or ACC; Enriquez-Geppert et al., 2010; Huster et al., 2013a), which might seem

874 at odds with an inhibitory role of rIFG. However, we argue these results indicate rIFG

875 interactions with pre-SMA are responsible for modulation of P300 timing. This

876 proposition is consistent with the results of our modeling of ERPs. DCM optimization

877 indicated rIFG governs inhibitory control through a top-down inhibitory coupling to pre-

878 SMA. Model comparison pointed to rIFG connections explicitly related to stopping

879 through a top-down, gain modulation over inhibitory interneurons in pre-SMA. This gain

880 modulation was dependent on descending rIFG activity, i.e., voltage. Additionally, rIFG

881 gain modulation was the only connection predictive of inhibitory success and SSRT

882 across TFUS conditions. The key implication of these results is that stopping potency

883 was causally related to rIFG activity levels directly increasing local inhibition in pre-SMA. 
884 These conclusions diverge from previous fMRI work (Duann et al., 2009; Rae et al., 885 2015) that found rIFG to pre-SMA coupling predicts inhibitory success and SSRT, but 886 also reported similarities across inhibitory and non-inhibitory tasks (Erika-Florence et al., 2014). Their interpretation was rIFG connectivity was a non-inhibitory process that signaled context-changes to pre-SMA. However, these studies only compared successful stopping against a baseline, without a pure comparison of stopping that we 890 induced with TFUS. regarding rIFG connectivity as driving inhibitory versus attentional control (Aron et al., 2016; Hampshire \& Sharp, 2015). We found rIFG to temporal and fronto-parietal preSMA to parietal connections interacted with inhibitory outcomes and TFUS. These ventral and fronto-parietal pathways, respectively, are typically associated with attentional reorienting (Corbetta et al., 2008; Vossel et al., 2014). Modulation of these pathways aligns with previous DCM studies explicitly manipulating top-down attention and stimulus expectancy (Auksztulewicz \& Friston, 2015; Fardo et al., 2017). However, because we found that neither connection predicted SSRT, we expect these putatively attentional pathway effects are secondary to inhibitory effects exerted by rIFG on preSMA. modulation, which we considered during model building. However, they differ in which neural population gain should be modulated by rIFG. Building upon neural models of 905 response inhibition (Lo et al., 2009; Logan et al., 2015) and circuit mechanisms (Roux \& 
907 tuning of local inhibitory interneuron gain through interareal connections. In contrast,

908 models underlying attention predicts top-down modulation or gain control over bottom-

909 up excitation (Desimone \& Duncan, 1995; Feldman \& Friston, 2010; Spratling, 2008).

910 DCM embeds excitatory gain through superficial pyramidal cells. Our formal model

911 comparison was consistent with a direct inhibitory control model. The winning model

912 involved rIFG modulating interneuron gain in pre-SMA (Fig $\mathbf{5 C}$ ). This finding has a

913 direct implication for explaining how rIFG could drive inhibition and how P300 onset

914 timing in pre-SMA could reflect the behavioral timing of inhibitory responses.

The interpretation of these results rests on the effect of neural gain operating as

a synaptic time-constant. Gain modulation can directly influence ERP timing of peaks

917 and decay rates. This suggest the P300 onset tracking of SSRT was directed by rIFG

918 activity levels that modulate inhibitory interneuron gain in pre-SMA. In turn, pre-SMA

919 gain alters the expression of P300 timing. Two caveats are worth noting here. First, our

920 model inferences are based on non-invasive measurements and require verification

921 from invasive electrophysiology. Second, given rIFG's substantial morphological

922 diversity and connectivity to other regions (Briggs et al., 2019) that are responsive to

923 attentional and perceptual processing factors (Erika-Florence et al., 2014; Levy \&

924 Wagner, 2011), we expect our results indicate an explicit inhibitory function operates

925 alongside other rIFG functions.

927 focused on sensor level analysis, some considering source activity (Enriquez-Geppert

928 et al., 2010), or invasive electrophysiology with limited cortical coverage (Swann et al.,

929 2012; Wessel et al., 2013). In contrast, a core focus of inhibition studies has been 
930 identifying exact locations of activated regions and thus employed fMRI. However, this approach filters fast neural responses, with connectivity methods being agnostic to

932 mechanistic instantiations of top-down and bottom-up interactions. We believe our

933 utilization of ERPs, online TFUS, and DCM for evoked responses is most appropriate

934 for differentiating bottom-up, top-down and gain effects driving evoked responses. This

935 stems from the fact that theories of inhibition or attention predict different gain

936 mechanisms, and these are instantiated in the microcircuit models within DCM (Bastos

937 et al., 2012). While a clear limitation is indeterminacy with source localization (Grech et

al., 2008), high temporal resolution is required to differentiate signals preceding

stopping-related events, e.g., SSRT. These methodological concerns are tempered by

pairing model-driven hypotheses with TFUS, because connectivity effects hold within

948 involves several processes, ranging from sensory cue detection, attention, performance 949 monitoring, and presumably explicit motor inhibition (Munakata, Herd, Chatham, Depue, 950 Banich, \& O’Reilly, 2011; Wessel \& Aron, 2017; Wiecki \& Frank, 2013). Our behavioral, 951 ERP, and connectivity results favor the framework wherein rIFG is directly involved in 952 explicit motor inhibition coinciding with attentional or sensory processing. The present 
953 application of high-spatial resolution TFUS and ERP modeling provides a neural and

954 methodological framework for testing other aspects of inhibitory and decision-making

955 function. A key question is whether these gain mechanisms are the basis for global

956 versus selective response inhibition. The efficacy of TFUS reported here also indicates

957 a possible neural intervention to pair with treatment for behavioral disorders stemming

958 from inhibitory response dysfunction, e.g., impulsivity or ADHD (Bari \& Robbins, 2013).

959

960

961

962

963

964

965

966

967

968

969

970

971

972

973

974

975

976

977

978

979

980 
981

982

983

984

985

986

987

988

989

990

991

992

993

994

995

996

997

998

999

1000

1001

1002

1003

1004

1005

1006

1007

1008

1009

1010

1011

1012

1013

1014

1015

1016

1017

1018

1019

1020

1021

1022

\section{References}

Alexander, W. H., \& Brown, J. W. (n.d.). Medial prefrontal cortex as an action-outcome predictor. https://doi.org/10.1038/nn.2921

Aron, A. R. (2006). Cortical and Subcortical Contributions to Stop Signal Response Inhibition: Role of the Subthalamic Nucleus. Journal of Neuroscience. https://doi.org/10.1523/JNEUROSCI.4682-05.2006

Aron, Adam R. (2011). From Reactive to Proactive and Selective Control: Developing a Richer Model for Stopping Inappropriate Responses. https://doi.org/10.1016/j.biopsych.2010.07.024

Aron, Adam R, Fletcher, P. C., Bullmore, E. T., Sahakian, B. J., \& Robbins, T. W. (2003). Stopsignal inhibition disrupted by damage to right inferior frontal gyrus in humans. 2002-2003. https://doi.org/10.1038/nn1003

Aron, Adam R, Herz, D. M., Brown, X. P., Forstmann, B. U., \& Zaghloul, K. (2016). Frontosubthalamic Circuits for Control of Action and Cognition. 36(45), 11489-11495. https://doi.org/10.1523/JNEUROSCI.2348-16.2016

Aron, Adam R, Robbins, T. W., \& Poldrack, R. A. (2014). Inhibition and the right inferior frontal cortex: one decade on. Trends in Cognitive Sciences, 18(4), 177-185. https://doi.org/10.1016/j.tics.2013.12.003

Aubry, J.-F., Tanter, M., Pernot, M., Thomas, J.-L., \& Fink, M. (2003). Experimental demonstration of noninvasive transskull adaptive focusing based on prior computed tomography scans. The Journal of the Acoustical Society of America. https://doi.org/10.1121/1.1529663

Auksztulewicz, R., \& Friston, K. (2015). Attentional enhancement of auditory mismatch responses: A DCM/MEG study. Cerebral Cortex. https://doi.org/10.1093/cercor/bhu323

Baillet, S., Rira, J. J., Main, G., Magin, J. F., Aubert, J., \& Ganero, L. (2001). Evaluation of inverse methods and head models for EEG source localization using a human skull phantom. Physics in Medicine and Biology. https://doi.org/10.1088/0031-9155/46/1/306

Bari, A., \& Robbins, T. W. (2013). Inhibition and impulsivity: Behavioral and neural basis of response control. In Progress in Neurobiology. https://doi.org/10.1016/j.pneurobio.2013.06.005

Bastos, A. M., Usrey, W. M., Adams, R. A., Mangun, G. R., Fries, P., \& Friston, K. J. (2012). Perspective Canonical Microcircuits for Predictive Coding. Neuron, 76(4), 695-711. https://doi.org/10.1016/j.neuron.2012.10.038

Bekker, E. M., Kenemans, J. L., Hoeksma, M. R., Talsma, D., \& Verbaten, M. N. (2005). The pure electrophysiology of stopping. International Journal of Psychophysiology. https://doi.org/10.1016/j.ijpsycho.2004.07.005

Belardinelli, P., Ortiz, E., Barnes, G., Noppeney, U., \& Preissl, H. (2012). Source Reconstruction Accuracy of MEG and EEG Bayesian Inversion Approaches. PLoS ONE, 7(12). https://doi.org/10.1371/journal.pone.0051985

Boehler, C. (2010). Pinning down response inhibition in the brain-conjunction analyses of the Stop-signal task4). Neuroimage, 52(1), 1621-1632.

https://doi.org/10.1016/j.neuroimage.2010.04.276 
1023

1024

1025

1026

1027

1028

1029

1030

1031

1032

1033

1034

1035

1036

1037

1038

1039

1040

1041

1042

1043

1044

1045

1046

1047

1048

1049

1050

1051

1052

1053

1054

1055

1056

1057

1058

1059

1060

1061

1062

1063

1064
Boucher, L., Palmeri, T. J., Logan, G. D., \& Schall, J. D. (2007). Inhibitory control in mind and brain: An interactive race model of countermanding saccades. Psychological Review. https://doi.org/10.1037/0033-295X.114.2.376

Briggs, R. G., Chakraborty, A. R., Anderson, C. D., Abraham, C. J., Palejwala, A. H., Conner, A. K., Pelargos, P. E., O'Donoghue, D. L., Glenn, C. A., \& Sughrue, M. E. (2019). Anatomy and white matter connections of the inferior frontal gyrus. Clinical Anatomy. https://doi.org/10.1002/ca.23349

Brown, H., Friston, K., \& Bestmann, S. (2011). Active inference, attention, and motor preparation. 2(September), 1-10. https://doi.org/10.3389/fpsyg.2011.00218

Brown, H. R., \& Friston, K. J. (2012). Dynamic causal modelling of precision and synaptic gain in visual perception - an EEG study. Neurolmage. https://doi.org/10.1016/j.neuroimage.2012.06.044

Chikazoe, J., Jimura, K., Hirose, S., Yamashita, K. I., Miyashita, Y., \& Konishi, S. (2009). Preparation to inhibit a response complements response inhibition during performance of a stop-signal task. Journal of Neuroscience. https://doi.org/10.1523/JNEUROSCI.364509.2009

Corbetta, M., Patel, G., \& Shulman, G. L. (2008). The Reorienting System of the Human Brain: From Environment to Theory of Mind. In Neuron. https://doi.org/10.1016/j.neuron.2008.04.017

Deco, G., \& Rolls, E. T. (2005). Neurodynamics of biased competition and cooperation for attention: A model with spiking neurons. Journal of Neurophysiology. https://doi.org/10.1152/jn.01095.2004

Delorme, A., \& Makeig, S. (2004). EEGLAB: An open source toolbox for analysis of single-trial EEG dynamics including independent component analysis. Journal of Neuroscience Methods, 134(1), 9-21. https://doi.org/10.1016/j.jneumeth.2003.10.009

Desimone, R., \& Duncan, J. (1995). Neural Mechanisms of Selective Visual Attention. Annual Review of Neuroscience. https://doi.org/10.1146/annurev.ne.18.030195.001205

Duann, J.-R., Ide, J. S., Luo, X., \& Li, C. R. (2009). Functional connectivity delineates distinct roles of the inferior frontal cortex and presupplementary motor area in stop signal inhibition. The Journal of Neuroscience: The Official Journal of the Society for Neuroscience, 29(32), 10171-10179. https://doi.org/10.1523/JNEUROSCI.1300-09.2009

Enriquez-Geppert, S., Konrad, C., Pantev, C., \& Huster, R. J. (2010). Conflict and inhibition differentially affect the N200/P300 complex in a combined go/nogo and stop-signal task. Neurolmage, 51(2), 877-887. https://doi.org/10.1016/J.NEUROIMAGE.2010.02.043

Erika-Florence, M., Leech, R., \& Hampshire, A. (2014). A functional network perspective on response inhibition and attentional control. Nature Communications. https://doi.org/10.1038/ncomms5073

Fardo, F., Auksztulewicz, R., Allen, M., Dietz, M. J., Roepstorff, A., \& Friston, K. J. (2017). Expectation violation and attention to pain jointly modulate neural gain in somatosensory cortex. Neurolmage. https://doi.org/10.1016/j.neuroimage.2017.03.041

Feldman, H., \& Friston, K. J. (2010). Attention, Uncertainty, and Free-Energy. Frontiers in Human Neuroscience, 4(December), 1-23. https://doi.org/10.3389/fnhum.2010.00215 
1065

1066

1067

1068

1069

1070

1071

1072

1073

1074

1075

1076

1077

1078

1079

1080

1081

1082

1083

1084

1085

1086

1087

1088

1089

1090

1091

1092

1093

1094

1095

1096

1097

1098

1099

1100

1101

1102

1103

1104

1105

1106

1107

Friston, K. J., Litvak, V., Oswal, A., Razi, A., Stephan, K. E., Van Wijk, B. C. M., Ziegler, G., \& Zeidman, P. (2016). Bayesian model reduction and empirical Bayes for group (DCM) studies. Neurolmage. https://doi.org/10.1016/j.neuroimage.2015.11.015

Grech, R., Cassar, T., Muscat, J., Camilleri, K. P., Fabri, S. G., Zervakis, M., Xanthopoulos, P., Sakkalis, V., \& Vanrumste, B. (2008). Review on solving the inverse problem in EEG source analysis. In Journal of NeuroEngineering and Rehabilitation. https://doi.org/10.1186/1743-0003-5-25

Greenhouse, I., \& Wessel, J. R. (2013). EEG signatures associated with stopping are sensitive to preparation. https://doi.org/10.1111/psyp.12070

Hampshire, A., Chamberlain, S. R., Monti, M. M., Duncan, J., \& Owen, A. M. (2010). The role of the right inferior frontal gyrus: inhibition and attentional control. Neuroimage, 50(3-3), 1313. https://doi.org/10.1016/J.NEUROIMAGE.2009.12.109

Hampshire, A., \& Sharp, D. J. (2015). Contrasting network and modular perspectives on inhibitory control. In Trends in Cognitive Sciences.

https://doi.org/10.1016/j.tics.2015.06.006

Hanes, D. P., Patterson, W. F., \& Schall, J. D. (1998). Role of frontal eye fields in countermanding saccades: Visual, movement, and fixation activity. Journal of Neurophysiology. https://doi.org/10.1152/jn.1998.79.2.817

Huster, R. J., Enriquez-geppert, S., Lavallee, C. F., Falkenstein, M., \& Herrmann, C. S. (2013). Electroencephalography of response inhibition tasks : Functional networks and cognitive contributions. International Journal of Psychophysiology, 87(3), 217-233. https://doi.org/10.1016/j.ijpsycho.2012.08.001

Huster, R. J., Enriquez-Geppert, S., Lavallee, C. F., Falkenstein, M., \& Herrmann, C. S. (2013a). Electroencephalography of response inhibition tasks: Functional networks and cognitive contributions. In International Journal of Psychophysiology. https://doi.org/10.1016/j.jpsycho.2012.08.001

Huster, R. J., Enriquez-Geppert, S., Lavallee, C. F., Falkenstein, M., \& Herrmann, C. S. (2013b). Electroencephalography of response inhibition tasks: Functional networks and cognitive contributions. https://doi.org/10.1016/j.ijpsycho.2012.08.001

Kenemans, J. L. (2015). Specific proactive and generic reactive inhibition. In Neuroscience and Biobehavioral Reviews. https://doi.org/10.1016/j.neubiorev.2015.06.011

Kibleur, A., Gras-Combe, G., Benis, D., Bastin, J., Bougerol, T., Chabardès, S., Polosan, M., \& David, O. (2016). Modulation of motor inhibition by subthalamic stimulation in obsessivecompulsive disorder. Translational Psychiatry, 6, 922. https://doi.org/10.1038/tp.2016.192

Kok, A., Ramautar, J. R., De Ruiter, M. B., Band, G. P. H., \& Ridderinkhof, K. R. (2004). ERP components associated with successful and unsuccessful stopping in a stop-signal task. Psychophysiology. https://doi.org/10.1046/j.1469-8986.2003.00127.x

Legon, W., Bansal, P., Tyshynsky, R., Ai, L., \& Mueller, J. K. (2018). Transcranial focused ultrasound neuromodulation of the human primary motor cortex. Scientific Reports. https://doi.org/10.1038/s41598-018-28320-1

Legon, W., Sato, T. F., Opitz, A., Mueller, J., Barbour, A., Williams, A., \& Tyler, W. J. (2014). Transcranial focused ultrasound modulates the activity of primary somatosensory cortex in humans. Nature Neuroscience. https://doi.org/10.1038/nn.3620 
1108

1109

1110

1111

1112

1113

1114

1115

1116

1117

1118

1119

1120

1121

1122

1123

1124

1125

1126

1127

1128

1129

1130

1131

1132

1133

1134

1135

1136

1137

1138

1139

1140

1141

1142

1143

1144

1145

1146

1147

1148

1149

1150

Levy, B. J., \& Wagner, A. D. (2011). Cognitive control and right ventrolateral prefrontal cortex: reflexive reorienting, motor inhibition, and action updating. Ann. N.Y. Acad. Sci. https://doi.org/10.1111/j.1749-6632.2011.05958.x

Lijffijt, M., Lane, S. D., Meier, S. L., Boutros, N. N., Burroughs, S., Steinberg, J. L., Gerard Moeller, F., \& Swann, A. C. (2009). P50, N100, and P200 sensory gating: Relationships with behavioral inhibition, attention, and working memory. Psychophysiology. https://doi.org/10.1111/j.1469-8986.2009.00845.x

Lo, C. C., Boucher, L., Paré, M., Schall, J. D., \& Wang, X. J. (2009). Proactive inhibitory control and attractor dynamics in countermanding action: A spiking neural circuit model. Journal of Neuroscience. https://doi.org/10.1523/JNEUROSCI.6164-08.2009

Logan, G. D., \& Cowan, W. B. (1984). On the ability to inhibit thought and action: A theory of an act of control. Psychological Review. https://doi.org/10.1037/0033-295X.91.3.295

Logan, G. D., Yamaguchi, M., Schall, J. D., \& Palmeri, T. J. (2015). Inhibitory control in mind and brain 2.0: Blocked-input models of saccadic countermanding. Psychological Review. https://doi.org/10.1037/a0038893

Lovett-Barron, M., Turi, G. F., Kaifosh, P., Lee, P. H., Bolze, F., Sun, X. H., Nicoud, J. F., Zemelman, B. V., Sternson, S. M., \& Losonczy, A. (2012). Regulation of neuronal input transformations by tunable dendritic inhibition. Nature Neuroscience. https://doi.org/10.1038/nn.3024

Mathôt, S., Schreij, D., \& Theeuwes, J. (2012). OpenSesame: An open-source, graphical experiment builder for the social sciences. In Behavior Research Methods. https://doi.org/10.3758/s13428-011-0168-7

Mattia, M., Spadacenta, S., Pavone, L., Quarato, P., Esposito, V., Sparano, A., Sebastiano, F., Di Gennaro, G., Morace, R., Cantore, G., \& Mirabella, G. (2012). Stop-event-related potentials from intracranial electrodes reveal a key role of premotor and motor cortices in stopping ongoing movements. Frontiers in Neuroengineering. https://doi.org/10.3389/fneng.2012.00012

Moran, R. (2015). Dynamic Causal Models for Human Electrophysiology: EEG, MEG, and LFPs. Brain Mapping, 625-628. https://doi.org/10.1016/B978-0-12-397025-1.00340-7

Moran, R. J., Campo, P., Symmonds, M., Stephan, K. E., Dolan, R. J., \& Friston, K. J. (2013). Free energy, precision and learning: The role of cholinergic neuromodulation. Journal of Neuroscience. https://doi.org/10.1523/JNEUROSCI.4255-12.2013

Mostofsky, S. H., \& Simmonds, D. J. (2008). Response inhibition and response selection: Two sides of the same coin. In Journal of Cognitive Neuroscience. https://doi.org/10.1162/jocn.2008.20500

Munakata, Y., Herd, S. A., Chatham, C. H., Depue, B. E., Banich, M. T., \& O'reilly, R. C. (2011). A unified framework for inhibitory control Opinion. Trends in Cognitive Sciences, 15(10), 453-459. https://doi.org/10.1016/j.tics.2011.07.011

Munakata, Y., Herd, S. A., Chatham, C. H., Depue, B. E., Banich, M. T., \& O'Reilly, R. C. (2011). A unified framework for inhibitory control. In Trends in Cognitive Sciences. https://doi.org/10.1016/j.tics.2011.07.011

Polich, J. (2007). Updating P300: An integrative theory of P3a and P3b. In Clinical Neurophysiology. https://doi.org/10.1016/j.clinph.2007.04.019 
1151

1152

1153

1154

1155

1156

1157

1158

1159

1160

1161

1162

1163

1164

1165

1166

1167

1168

1169

1170

1171

1172

1173

1174

1175

1176

1177

1178

1179

1180

1181

1182

1183

1184

1185

1186

1187

1188

1189

1190

1191

1192

1193
Rae, C. L., Hughes, L. E., Anderson, M. C., \& Rowe, J. B. (2015). The prefrontal cortex achieves inhibitory control by facilitating subcortical motor pathway connectivity. Journal of Neuroscience. https://doi.org/10.1523/JNEUROSCI.3093-13.2015

Roux, L., \& Buzsáki, G. (2015). Tasks for inhibitory interneurons in intact brain circuits. In Neuropharmacology. https://doi.org/10.1016/j.neuropharm.2014.09.011

Rushworth, M. F. S., Walton, M. E., Kennerley, S. W., \& Bannerman, D. M. (2004). Action sets and decisions in the medial frontal cortex. In Trends in Cognitive Sciences. https://doi.org/10.1016/j.tics.2004.07.009

Sharp, D. J., Bonnelle, V., De Boissezon, X., Beckmann, C. F., James, S. G., Patel, M. C., \& Mehta, M. A. (2010). Distinct frontal systems for response inhibition, attentional capture, and error processing. Proceedings of the National Academy of Sciences of the United States of America. https://doi.org/10.1073/pnas.1000175107

Spratling, M. W. (2008). Reconciling predictive coding and biased competition models of cortical function. Frontiers in Computational Neuroscience. https://doi.org/10.3389/neuro.10.004.2008

Swann, N. C., Cai, W., Conner, C. R., Pieters, T. A., Claffey, M. P., George, J. S., Aron, A. R., \& Tandon, N. (2011). Neurolmage Roles for the pre-supplementary motor area and the right inferior frontal gyrus in stopping action: Electrophysiological responses and functional and structural connectivity. https://doi.org/10.1016/j.neuroimage.2011.09.049

Swann, N. C., Cai, W., Conner, C. R., Pieters, T. A., Claffey, M. P., George, J. S., Aron, A. R., \& Tandon, N. (2012). "Roles for the pre-supplementary motor area and the right inferior frontal gyrus in stopping action: electrophysiological responses and functional and structural connectivity." Neurolmage, 59(3), 2860-2870. https://doi.org/10.1016/j.neuroimage.2011.09.049

Thiele, A., \& Bellgrove, M. A. (2018). Neuromodulation of Attention. In Neuron. https://doi.org/10.1016/j.neuron.2018.01.008

Treeby, B. E., \& Cox, B. T. (2010). k-Wave: MATLAB toolbox for the simulation and reconstruction of photoacoustic wave fields. Journal of Biomedical Optics. https://doi.org/10.1117/1.3360308

Vossel, S., Geng, J. J., \& Fink, G. R. (2014). Dorsal and ventral attention systems: Distinct neural circuits but collaborative roles. Neuroscientist. https://doi.org/10.1177/1073858413494269

Wessel, J. R., \& Aron, A. R. (2015). It's not too late: The onset of the frontocentral P3 indexes successful response inhibition in the stop-signal paradigm. Psychophysiology, 52(4), 472480. https://doi.org/10.1111/psyp.12374

Wessel, J. R., \& Aron, A. R. (2017). On the Globality of Motor Suppression: Unexpected Events and Their Influence on Behavior and Cognition. In Neuron. https://doi.org/10.1016/j.neuron.2016.12.013

Wessel, J. R., Conner, C. R., Aron, A. R., \& Tandon, N. (2013). Chronometric electrical stimulation of right inferior frontal cortex increases motor braking. Journal of Neuroscience. https://doi.org/10.1523/JNEUROSCI.3468-13.2013

Wiecki, T. V, \& Frank, M. J. (2013). A Computational Model of Inhibitory Control in Frontal Cortex and Basal Ganglia. 120(2), 329-355. https://doi.org/10.1037/a0031542 
bioRxiv preprint doi: https://doi.org/10.1101/649665; this version posted April 24, 2020. The copyright holder for this preprint (which was not certified by peer review) is the author/funder, who has granted bioRxiv a license to display the preprint in perpetuity. It is made available under aCC-BY-NC-ND 4.0 International license.

1195 